\title{
Structures and Components in Galaxy Clusters: Observations and Models
}

\author{
A.M. Bykov ${ }^{1,2,3}$ - E.M. Churazov ${ }^{4,5}$ - C. Ferrari' ${ }^{6}$. \\ W.R. Forman ${ }^{7}$. J.S. Kaastra ${ }^{8}$ - U. Klein ${ }^{9}$. \\ M. Markevitch ${ }^{10}$. J. de Plaa $^{8}$
}

Received: 23 November 2014 / Accepted: 22 December 2014 / Published online: 4 February 2015

CC Springer Science+Business Media Dordrecht 2015

\begin{abstract}
Clusters of galaxies are the largest gravitationally bounded structures in the Universe dominated by dark matter. We review the observational appearance and physical models of plasma structures in clusters of galaxies. Bubbles of relativistic plasma which are inflated by supermassive black holes of AGNs, cooling and heating of the gas, large scale plasma shocks, cold fronts, non-thermal halos and relics are observed in clusters. These con-
\end{abstract}

A.M. Bykov

byk@astro.ioffe.ru

E.M. Churazov

churazov@mpa-garching.mpg.de

C. Ferrari

chiara.ferrari@oca.eu

W.R. Forman

forman@cfa.harvard.edu

J.S. Kaastra

j.kaastra@sron.nl

U. Klein

uklein@astro.uni-bonn.de

M. Markevitch

maxim.markevitch@nasa.gov

J. de Plaa

J.de.Plaa@sron.nl

1 A.F. Ioffe Institute for Physics and Technology, 194021, St. Petersburg, Russia

2 St. Petersburg State Politecnical University, St. Petersburg, Russia

3 International Space Science Institute, Bern, Switzerland

4 Max Planck Institute for Astrophysics, Karl-Schwarzschild-Str. 1, 85741 Garching, Germany

5 Space Research Institute (IKI), Profsoyuznaya 84/32, Moscow 117997, Russia

6 Laboratoire Lagrange, UMR7293, Université de Nice Sophia-Antipolis, CNRS, Observatoire de la Côte d'Azur, Boulevard de l'Observatoire, 06300 Nice, France 
stituents are reflecting both the formation history and the dynamical properties of clusters of galaxies. We discuss X-ray spectroscopy as a tool to study the metal enrichment in clusters and fine spectroscopy of Fe X-ray lines as a powerful diagnostics of both the turbulent plasma motions and the energetics of the non-thermal electron populations. The knowledge of the complex dynamical and feedback processes is necessary to understand the energy and matter balance as well as to constrain the role of the non-thermal components of clusters.

Keywords Clusters of galaxies - Radiation mechanisms: non-thermal $\cdot$ Radio continuum X-rays: galaxies: clusters

\section{Introduction}

Clusters of galaxies grow by gravitational collapse to the most massive objects of the Universe. While the total mass is dominated by dark matter $(\sim 80 \%)$, there is a significant baryonic contribution of $20 \%$ and the processes where baryons are involved prominently determine the evolutionary physics and the observational appearance of clusters.

In this paper we focus on the baryonic component of clusters and in particular on the hot gas that, with its thermal and non-thermal constituents, comprises the majority of these baryons. Cluster galaxies are embedded in this hot intracluster medium (ICM), but represent a small fraction of both the volume and the baryonic mass.

For most clusters, the hot gas reaches temperatures of $10^{7}-10^{8} \mathrm{~K}$. At the low temperature end, below temperatures of about $2 \times 10^{7} \mathrm{~K}$, it is more common to speak about groups of galaxies rather than clusters of galaxies, but the transition is of course smooth.

From an X-ray perspective, clusters are found in two variants: those with a cool core and those lacking such a core. In the cool core clusters the density of the gas in the center reaches values of $\sim 10^{-2} \mathrm{~cm}^{-3}$ (that is, for instance, only $1 \%$ of the typical density of the interstellar medium of a galaxy). The density decreases rapidly towards the outskirts down to levels of order $10^{-4} \mathrm{~cm}^{-3}$ or less.

The density in the cool cores is high enough to cause significant cooling over cosmological time scales through thermal bremsstrahlung emission observed in X-rays. Without any heating mechanism, this gas would cool down further and do form stars. However, it appears that active nuclei in the core of the dominant cluster galaxies can emit so much power that the associated heating compensates the cooling of the central gas. This leads to a physically interesting but complex feedback loop between the central supermassive black hole and the cluster gas. These processes and the associated plasma structures will be discussed in this review.

Clusters are by no means static entities, they still grow. Large scale violent processes occur, like cluster mergers or, more frequently, the capture of groups or individual galaxies by massive systems. Due to the supersonic velocities involved, shocks are produced at various locations within colliding clusters. This leads to local heating, particle acceleration and modification of the magnetic fields, and we will also discuss these processes.

7 Harvard Smithsonian Center for Astrophysics, 60 Garden Street, Cambridge, MA 02138, USA

8 SRON Netherlands Institute for Space Research, Sorbonnelaan 2, 3584 CA Utrecht, Netherlands

9 Argelander-Institut für Astronomie, University of Bonn, Bonn, Germany

10 Astrophysics Science Division, NASA/Goddard Space Flight Center, Greenbelt, MD 20771, USA 
Another part of the bulk motions may cascade downwards in scale in the form of turbulence, that represents a contribution to the total thermal pressure of the order of 5-15\% and is stronger in the outer parts of clusters (see for review Kravtsov and Borgani 2012; Dolag et al. 2008). In these external regions, infalling galaxies and groups will give rise to density inhomogeneities which, due to the relatively low density in cluster outskirts, take a long time to disappear.

Mixing of merging components also leads to interesting processes at the interface between cold and hot gas. Further, when galaxies are being captured by a cluster, they may lose their chemically enriched gas to the intracluster medium by ram pressure or interaction with other galaxies. The ICM itself is a rich archive of the past chemical history of the cluster: it contains information on the distribution of stars and the relative frequencies of specific subclasses.

Clusters, that are the largest gravitationally bound systems in the Universe, form a rich and living laboratory to study all kinds of processes that shape their appearance. Our review is devoted to discussion of plasma structures of different scales and origin in clusters of galaxies. More general discussion and many important ideas about the evolution and cosmological importance of clusters of galaxies can be found in recent reviews (see e.g. Böhringer and Werner 2010; Allen et al. 2011; Kravtsov and Borgani 2012; Planelles et al. 2014, and references therein).

\section{AGNs in Galaxy Clusters. Feedback Processes}

The gas in the cores of galaxy clusters has a radiative cooling time of the order of $10^{9}$ years or less, opening the possibility of forming an extremely massive central galaxy. This occurs in numerical simulations with radiative cooling, but is generally not observed in nature, with a few observed exceptions, such as, e.g., the Phoenix cluster (McDonald et al. 2012). Instead, observations suggest that the mechanical energy released by a central AGN regulates the thermal state of the gas, preventing it from catastrophic cooling. Bubbles of relativistic plasma are inflated by a supermassive black hole and rise buoyantly through the gaseous atmosphere, leading to a number of spectacular phenomena such as expanding shocks, $\mathrm{X}$ ray dim and radio bright cavities, old and "dead" cavities and filaments in the wakes of the rising bubbles formed by entrained low entropy gas.

Simple arguments based on the energy content of bubbles and their life-time show that the amount of mechanical energy supplied by AGNs matches approximately the gas cooling losses in objects vastly different in size and luminosity. This hints at some form of selfregulation of the AGN power. How the mechanical energy, provided by the AGN, is dissipated depends on the ICM microphysics (e.g. magnetic fields, viscosity, conduction etc.). However it is plausible that close to $100 \%$ of the mechanical energy is eventually dissipated in the cluster core, regardless of the particular physical process involved.

AGN feedback is plausibly a key process for the formation of massive ellipticals at $z \sim$ $2-3$, as suggested by the correlation of galaxy bulge properties and the mass of the SMBH (Ferrarese and Merritt 2000; Gebhardt et al. 2000). Galaxy clusters offer us a zoomed view on this process at $z \sim 0$. Three pre-requisites are needed for this scenario to work (i) a hot gaseous atmosphere in the galaxy is present, (ii) the black hole is sufficiently massive and (iii) a large fraction of AGN energy is in mechanical form. The latter depends critically on the physics of accretion, in particular, on the transition of the SMBH energy output from the radiation-dominated mode to the mechanically-dominated mode when the accretion rate drops below a fraction of the Eddington value (e.g., Churazov et al. 2005). Given that the 
coupling constant of these two forms of energy output with the ICM can differ by a factor of $10^{4}-10^{5}$, this change in the accretion mode may explain the switch of a SMBH (and its parent galaxy) from the QSO-type behavior and an intense star formation epoch to the radiatively inefficient AGN and essentially passive evolution of the parent galaxy.

Below we briefly outline only most general features of the AGN Feedback model in galaxy clusters and do not discuss results from numerical simulations. Extended reviews on the AGN Feedback in clusters can be found in, e.g., McNamara and Nulsen (2007), Fabian (2012).

\subsection{Cluster Cores Without AGN Feedback}

The radiative cooling time of the gas in the central parts of rich galaxy clusters $\left(t_{\text {cool }}=\right.$ $\frac{\gamma}{\gamma-1} \frac{n k T}{n^{2} \Lambda(T)} \sim$ few $10^{8}-10^{9} \mathrm{yr}$ ) is shorter than the Hubble time (e.g., Lea 1976; Cowie and Binney 1977; Fabian and Nulsen 1977). Here $n$ and $T$ are the density and temperature of the ICM, respectively, $\gamma$ is the adiabatic index and $\Lambda(T)$ is the radiative cooling function. Since $t_{\text {cool }} \propto 1 / n$, the cooling time is short in the center, but rapidly increases with radius. The radius $r_{\text {cool }}$ where $t_{\text {cool }} \sim t_{\text {Hubble }}$ is usually referred to as the cooling radius. Without an external source of energy, the gas inside $r_{\text {cool }}$ must cool and flow towards the center, forming a so-called "cooling flow" (see Fabian 1994, for a review of the scenario without AGN feedback). Observations (e.g., Peterson and Fabian 2006; David et al. 2001), however, suggest that the net rate of gas cooling to low temperatures is a small fraction $(\sim 10 \%$ or below) of the straightforward estimate:

$$
\dot{M}_{\text {cool }}=\frac{L_{\text {cool }}}{\frac{\gamma}{\gamma-1} k T} \mu m_{\mathrm{p}} \sim 10^{2}-10^{3} M_{\odot} \mathrm{yr}^{-1},
$$

where $L_{\text {cool }}$ is the total cooling rate within $r_{\text {cool }}, \mu$ is the mean particle atomic weight. This implies that a source of heat is needed to offset ICM cooling losses. In the late 90's and early 2000's it became clear that a supermassive black hole sitting at the center of the dominant cluster galaxy could operate as such source.

The above "cooling flow" problem applies not only to rich clusters and groups, but also to individual hot gas rich galaxies (e.g., Thomas et al. 1986). The fact that these galaxies do not show significant star formation argues for feedback from these low mass systems (some with SMBH masses as large as those in central galaxies in rich clusters) up to the most massive and X-ray luminous clusters.

\subsection{Evidence for AGN Mechanical Feedback and Its Energetics}

Massive ellipticals at the cores of rich clusters host black holes with masses larger than $10^{9} M_{\odot}$. In terms of energetics, such black holes, accreting at the Eddington rate, could release up to $10^{47} \mathrm{erg} \mathrm{s}^{-1}$ — much more than needed to reheat the gas. However, we do not find extremely bright AGNs in nearby clusters, and the coupling of radiation to the fully ionized ICM (via Compton scattering) is weak. Based on radio observations, Pedlar et al. (1990) argued that mechanical power of jets in NGC1275 (dominant galaxy in the Perseus cluster) is likely much higher than their radiative power and it might be comparable to the gas cooling losses. For less massive systems Tabor and Binney (1993) and Binney and Tabor (1995) suggested that the mechanical power of jets may have a strong impact on the thermal state of the gas in the central region. But it was only the combination of X-ray and radio data that convincingly demonstrated a crucial role of AGN feedback in cluster cores. 


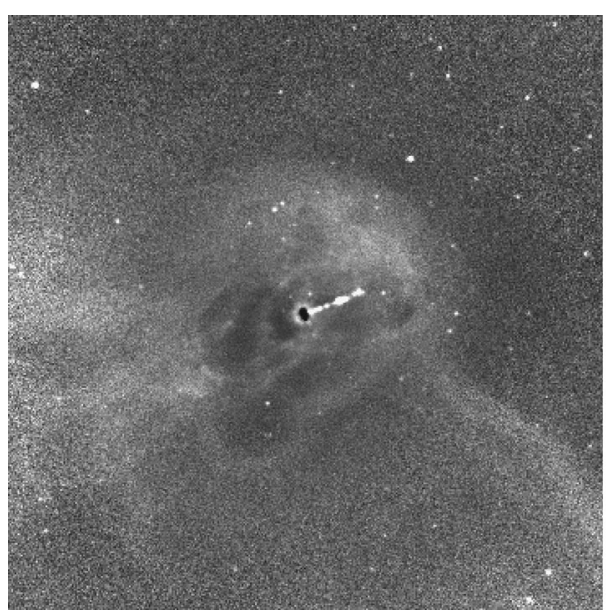

Fig. 1 X-ray (Forman et al. 2007) and $6 \mathrm{~cm}$ radio (Hines et al. 1989) images of the M87 core $\left(3^{\prime} \times 3^{\prime}\right)$. The X-ray image (left) was flat-fielded to emphasize various non-axisymmetric features. Images are shown to scale, centered at the supermassive black hole with a kpc scale jet going to the NW. X-ray cavities, matching the $6 \mathrm{~cm}$ image of the inner radio lobes are clearly seen in the left image

By now, Chandra and XMM-Newton found signs of AGN/ICM interactions in a large fraction of relaxed clusters (e.g., McNamara et al. 2000; Dunn and Fabian 2006; Rafferty et al. 2006; Hlavacek-Larrondo et al. 2012; Bîrzan et al. 2012). But clear evidence of this process in the Perseus cluster and in M87/Virgo had previously been seen in ROSAT images (Boehringer et al. 1993; Bohringer et al. 1995). Based on these sketchy X-ray and radio images, the basic features of the mechanical feedback model and its energetics, inspired by analogy with powerful atmospheric explosions, were outlined (Churazov et al. 2000) just before Chandra and XMM-Newton launch.

This impact of the SMBH mechanical power on the ICM can be best illustrated for M87-the X-ray brightest elliptical galaxy in the nearby Virgo cluster. While M87/Virgo is not a very luminous system $\left(L_{\text {cool }}\right.$ is of the order $10^{43} \mathrm{erg} \mathrm{s}^{-1}$, e.g. Peres et al. 1998, Owen et al. M87), its proximity (distance $\sim 16 \mathrm{Mpc}$ ) offers an exquisitely detailed view on the processes in the very core of the galaxy (e.g. Forman et al. 2005, 2007; Million et al. 2010; Werner et al. 2010).

Shown in Fig. 1 are the X-ray and $6 \mathrm{~cm}$ radio images of M87 (central $\left.3^{\prime} \times 3^{\prime}\right)$. Synchrotron emission for a jet coming from the SMBH is clearly visible. Except for the jet, the X-ray image is dominated by the thermal emission of the optically thin plasma at a temperature 1-2 keV (unambiguously proven by the observed spectra). The radio image on the right shows synchrotron emission from the jet and radio lobes filled with relativistic plasma. The radio-bright lobes at $6 \mathrm{~cm}$ nicely correspond to the $\mathrm{X}$-ray dim regions that are defined by $\mathrm{X}$-ray bright shells, suggesting that thermal plasma is displaced by the relativistic plasma within the central $1^{\prime}$ from the core. A minimum energy, required to (slowly) inflate a bubble of a given volume at a constant pressure is the enthalpy:

$$
E_{\mathrm{bub}}=\frac{\gamma}{\gamma-1} P V
$$

where $\gamma$ is the adiabatic index of the fluid inside the bubble $(\gamma=4 / 3$ and $5 / 3$ for monoatomic relativistic and non-relativistic fluids respectively), $P$ is the ICM pressure and $V$ is 
the volume. This gives a lower limit on the amount of mechanical energy produced by the SMBH. Since we do not see evidence for a very strong shock surrounding the lobes (see, however, below), the true amount of energy should not be far from this lower limit.

To evaluate the mechanical power of the AGN, we need to estimate the life-time of the bubbles $t_{\text {bub }}$. The simplest recipe (Churazov et al. 2000) comes from the analysis of the buoyancy driven evolution of the bubble. The importance of buoyancy for the radio lobes, filled with relativistic plasma, was pointed out by Gull and Northover (1973). Comparing the expansion velocity (which depends on the AGN power and the size of the bubble) and the buoyant rise time (which depends on the gravity and the size of the bubble) one gets an upper limit on the life time of the bubble. For a steady AGN power $L_{\mathrm{AGN}}$ deposited into a small volume, the initial expansion of the bubble is supersonic, but it slows as the bubble grows. Soon after the expansion velocity becomes subsonic, the bubble is deformed by the Rayleigh-Taylor instability and rises under the action of buoyancy. The terminal velocity of the rising bubble $v_{\text {rise }}$ is set by the balance of the ram pressure (assuming low viscosity of the ICM)and the buoyancy force:

$$
g \frac{4}{3} \pi r^{3} \rho_{\mathrm{gas}} \approx C \pi r^{2} \rho_{\mathrm{gas}} v^{2},
$$

where $g$ is the gravitational acceleration, $r$ is the bubble radius, $\rho_{\text {gas }}$ is the ICM density and $C$ is a dimensionless constant of order unity. Thus $v_{\text {rise }} \sim \sqrt{g r}$. At the same, time AGN activity drives the expansion of the bubble with the velocity $v_{\exp }$ set by the AGN power $L_{\mathrm{AGN}}$, e.g., from Eq. (2) $v_{\exp } \sim \frac{\gamma-1}{\gamma} \frac{L_{\mathrm{AGN}}}{4 \pi P r^{2}}$, provided that the expansion velocity is subsonic. The condition $v_{\exp } \gtrsim v_{\text {rise }}$ sets the lower limit on the AGN power needed to ignore the role of buoyancy. For M87 the size of the inner lobes suggests the jet mechanical power $L_{\mathrm{AGN}} \sim \frac{\gamma}{\gamma-1} \frac{4 \pi P r^{2}}{\sqrt{g r}} \sim$ few $10^{43} \mathrm{erg} \mathrm{s}^{-1}$. Of course such estimates (and various modifications e.g., McNamara and Nulsen 2007) are only accurate to within a factor of a few. Nevertheless, it is clear that mechanical input of the SMBH is sufficient to offset gas cooling losses, i.e. $L_{\mathrm{AGN}} \sim 10^{43} \mathrm{erg} \mathrm{s}^{-1} \sim L_{\text {cool }}$.

One can expect that the initial phase of the bubble expansion is supersonic and it will drive a shock into the ICM. As the expansion slows, the shock weakens and moves ahead of the expanding boundary. These shocks offer yet another way to measure the AGN power. In M87 we see at least two generations of shocks (Forman et al. 2005, 2007) at $0.6^{\prime}$ and $2.7^{\prime}$ from the center (Fig. 2). The hard emission (3.5-7.5 keV) shows a ring of emission with an outer radius ranging from $2.5^{\prime}$ to $2.85^{\prime}(11.6-13.3 \mathrm{kpc})$. This ring of hard emission provides an unambiguous signature of a weak shock. The gas temperature in the ring rises from $\sim 2.0 \mathrm{keV}$ to $\sim 2.4 \mathrm{keV}$ implying a Mach number of $\mathcal{M} \sim 1.2$ (shock velocity $v=880 \mathrm{~km} \mathrm{~s}^{-1}$ for a $2 \mathrm{keV}$ thermal gas). At the shock, the density jump is 1.33 which yields a Mach number of 1.22, consistent with that derived from the temperature jump. The total energy needed to drive this shock can be readily estimated from 1D hydro simulations $E \sim 5 \times 10^{57} \mathrm{erg}$. The age of the outburst that gave rise to the shock is $\sim 12 \mathrm{Myr}$. If we divide the energy of the outburst by the age of the shock we get a mean energy release $\sim 10^{43} \mathrm{erg} \mathrm{s}^{-1}$ — broadly in agreement with the ICM cooling losses.

\subsection{Dissipation of Mechanical Energy}

Once the bubble is detached from the central source, its evolution is governed by buoyancy. During the rise the bubble may transform into a toroidal structure as the "ear-like" structure in M87 (see Figs. 3 and 4) which resembles a mushroom formed by a powerful atmospheric explosion. As in case of the atmospheric explosion, the bubble is able to entrain 

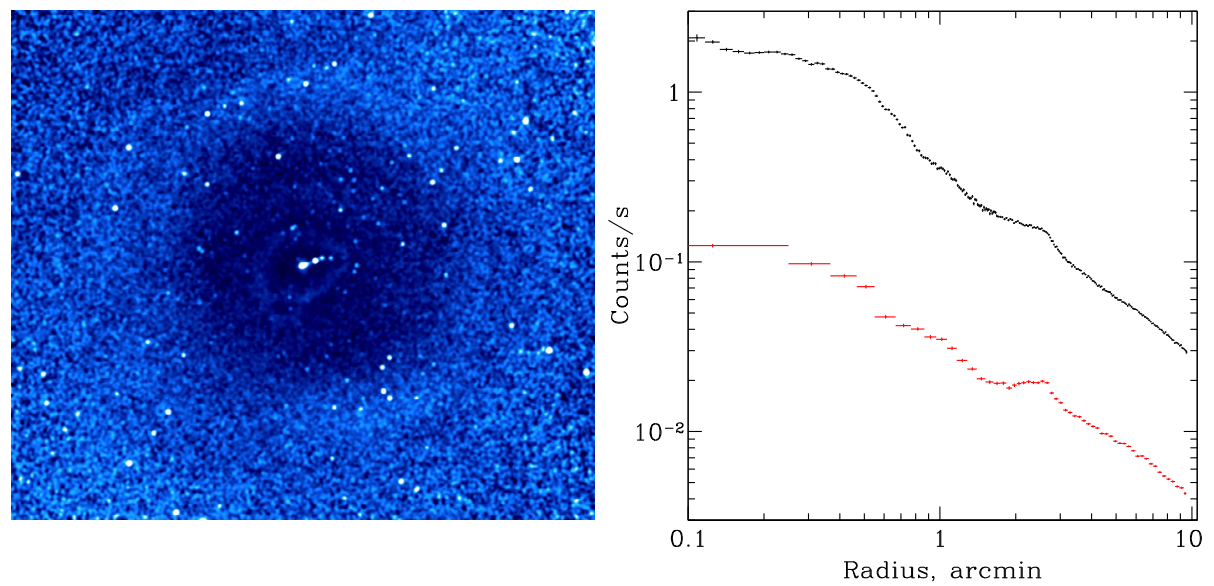

Fig. 2 Left: Chandra image of M87 $\left(\sim 7^{\prime} \times 7^{\prime}\right)$ in the 3.5-7.5 keV energy band divided by a spherically symmetric model. This energy band shows pressure variations in the gas (Forman et al. 2007). A nearly perfect ring at $\sim 2.75^{\prime}(12.8 \mathrm{kpc})$ is clearly seen. This is a characteristic signature of a shock, driven by an outburst from the central SMBH. Right: the surface brightness profiles (Forman et al. 2007) in the 1.2-2.5 keV (upper curve) and 3.5-7.5 keV (lower curve) bands show a prominent feature at $2-3^{\prime}$, along with a fainter feature at $0.6^{\prime}$
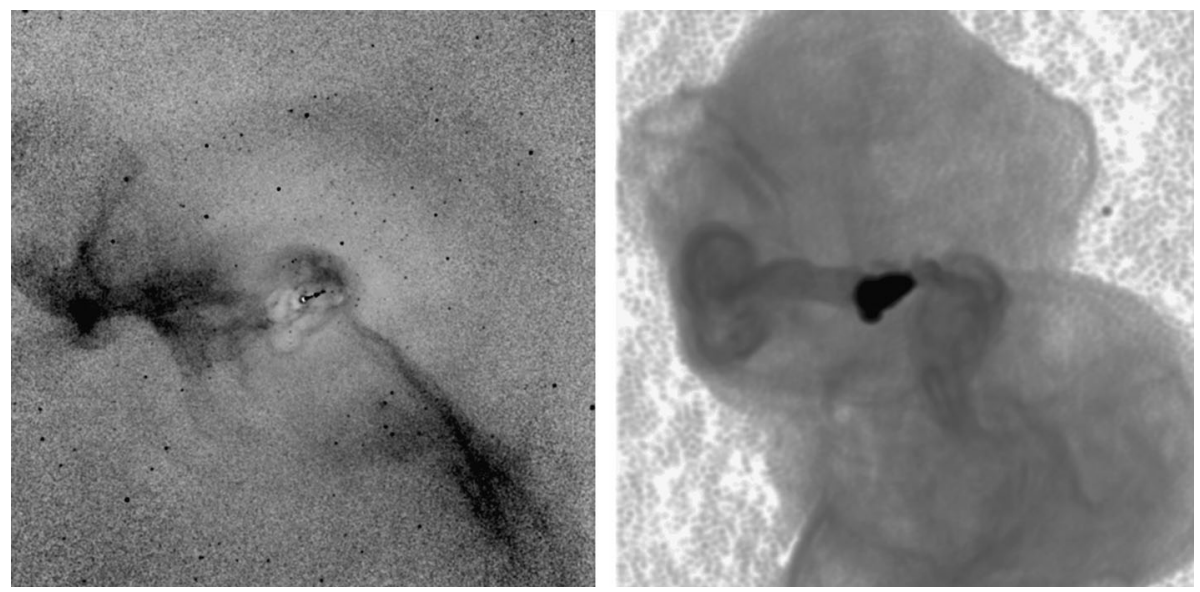

Fig. 3 X-ray (Forman et al. 2007) and $90 \mathrm{~cm}$ radio (Owen et al. M87) images (right) of the core of M87 $\left(8^{\prime} \times 8^{\prime}\right)$. Filaments of cool gas are entrained by the buoyantly rising bubbles

large amounts of ambient gas from the core of cluster and transport it to large distance from the cluster center (e.g., Churazov et al. 2001; Fabian et al. 2003; Werner et al. 2010).

Note that adiabatic expansion of rising bubbles leads to a rapid decrease of the radio emission, since both the magnetic field strength, and the density and energy of relativistic particles are decreasing at the same time. This decrease is especially strong if the aging break in the distribution of electrons is brought by adiabatic expansion into the observable frequency range. Thus, unless there is continuous reacceleration of electrons, the radio bright bubbles should evolve into a radio dim objects. Since the pressure support inside the bubble could still come from magnetic fields and low energy electrons and protons (Lorentz factor 


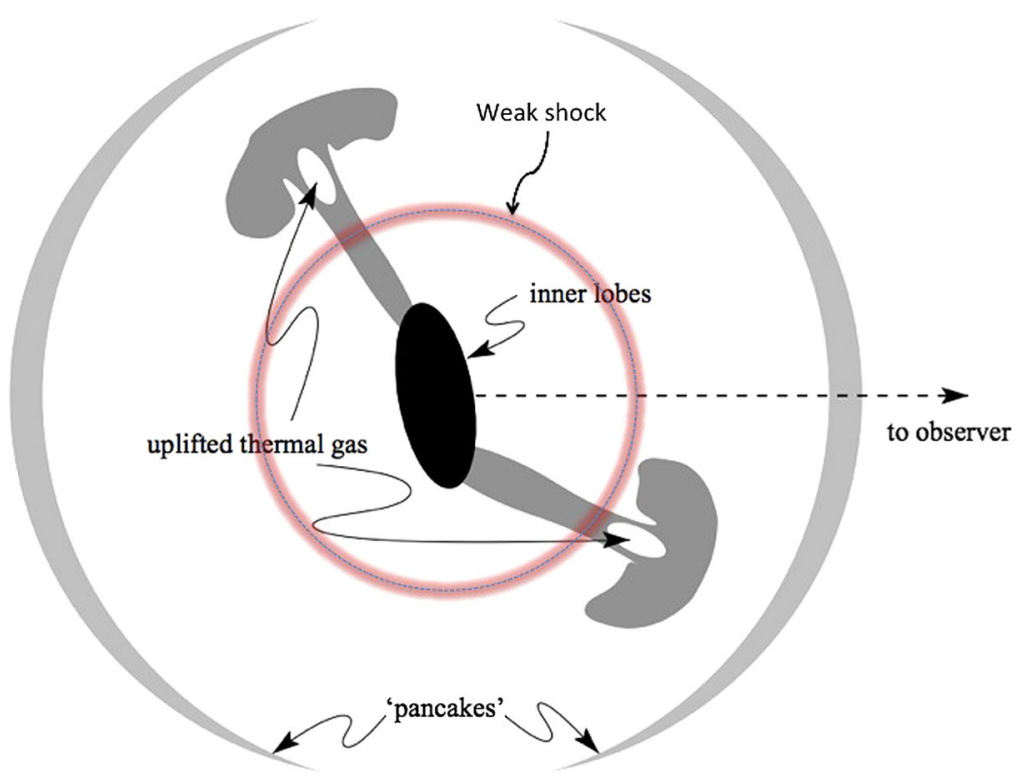

Fig. 4 Schematic picture of major signs of AGN/ICM interaction (adapted and modified from Churazov et al. 2001), inspired by analogy with mushroom clouds produced by powerful atmospheric explosions. The black region in the center denotes the inner radio lobes, driven by the SMBH mechanical power. The circular structure is a weak shock wave produced by these inner lobes. Gray "mushrooms" correspond to the buoyant bubbles already transformed into tori, and the gray lens-shaped structures are the pancakes formed by the older bubbles (cf. Fig. 3)

of 1000 or lower), the bubble is still seen as an X-ray cavity, but is very dim in radio. Such ghost bubbles are believed to be widespread in the cluster cores and one can expect many of them to be detected with a new generation of a low frequency instruments (Enßlin and Heinz 2002).

The bubble rise velocity $v_{\text {rise }}$ is smaller than the ICM sound speed, and it is much smaller than the sound speed of the relativistic fluid inside the bubble. Adiabatic expansion of the rising bubble implies that its enthalpy is decreasing according to the ambient gas pressure $H=\frac{\gamma}{\gamma-1} P V \propto P^{\frac{\gamma-1}{\gamma}}$. This means that after crossing a few pressure scale heights, much of the energy originally stored as the enthalpy of the relativistic bubble is transferred to the gas. Subsonic motion with respect to the ambient gas guarantees that only a fraction of this energy is "lost" as sound waves, which may leave the cluster core. Thus we can conclude that a fraction of energy of order unity is transferred to the ICM. This leads to the conjecture that essentially all of the mechanical energy is dissipated in the cluster core, which acts as a calorimeter of AGN activity (Churazov et al. 2002).

Details of the dissipation process depend sensitively on the properties of the ICM. For instance, this energy could drive turbulent motions in the wake of the rising bubble and excite gravity waves (e.g., Churazov et al. 2001; Omma et al. 2004). These motions will eventually dissipate into heat. Alternatively (if the ICM viscosity is high), the energy can be dissipated directly in the flow around the bubble. However in either case, the energy does not escape from the cluster core.

An interesting recent development came from the analysis of X-ray surface brightness fluctuations in the Perseus and M87/Virgo clusters (Zhuravleva et al. 2014). If the observed fluctuations are interpreted as weak perturbations of a nearly hydrostatic cluster atmosphere, 
Fig. 5 Turbulent heating versus gas cooling rates in the Perseus and Virgo cores. Shaded regions show the heating and cooling rates estimated at different distances from the cluster center. The size of each region reflects estimated statistical and stochastic uncertainties. (Adapted from Zhuravleva et al. 2014)

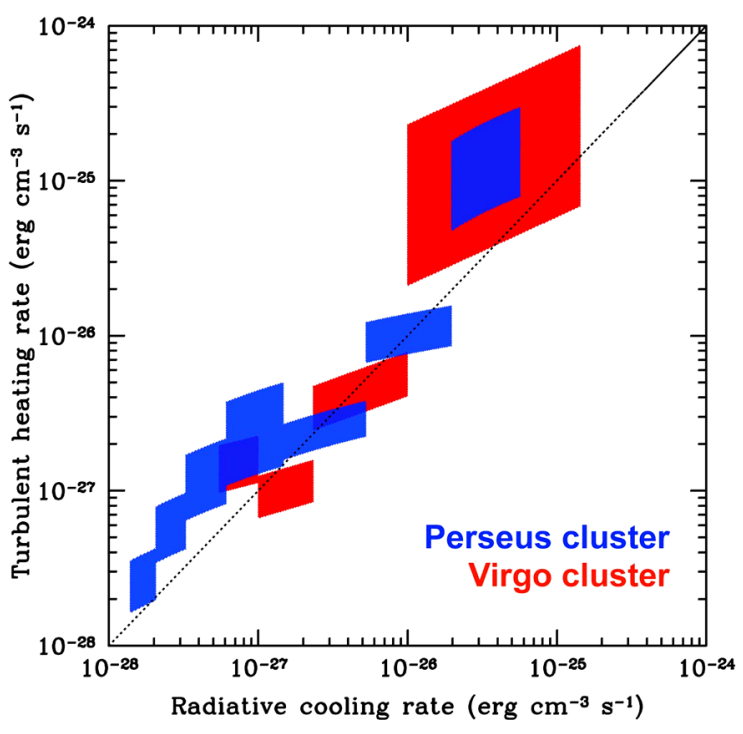

then one can link their amplitude to the characteristic gas velocities at different spatial scales. Since in the canonical Kolmogorov turbulence the energy flow is constant across the inertial range, it is sufficient to know the velocity $V$ at one scale $l$ (within the inertial range) to estimate the dissipation rate as $Q_{\text {turb }} \sim \rho \frac{V^{3}}{l}$. Applying this approach to Perseus and M87/Virgo leads to a tantalizing conclusion that the turbulent dissipation approximately matches the gas cooling rate in these clusters (Fig. 5). While a number of assumptions enter these calculations, the result is encouraging. Future ASTRO-H measurements of the gas velocities in these clusters should be able to verify these findings.

A substantial fraction of energy released by the SMBH can go into quasi-spherical sound waves propagating through the ICM. Unlike strong shocks the dissipation of the energy carried by sound waves depends on the ICM microphysics, but it is plausible that their energy will be dissipated before the wave leaves the core of the cluster (Fabian et al. 2006). The attractiveness of this model is that the energy can be evenly distributed over large regions. Whether sound waves provide the dominant source of heat to the ICM depends critically on what fraction of AGN energy goes to sound waves. In M87 the fraction of energy, which went into the weak shock is $\lesssim 25 \%$ (Forman et al. 2007).

\subsection{Self-regulation}

Similar signs of SMBH-ICM interaction are observed for objects having vastly different sizes and luminosities. Two examples are shown in Fig. 6-these are $3^{\prime} \times 3^{\prime} \mathrm{X}$-ray images of the elliptical galaxy NGC5813 (Randall et al. 2011) and the Perseus cluster (Fabian et al. 2000). In each case, X-ray cavities of approximately the same angular size are clearly seen. The distances to NGC5813 and the Perseus cluster are 32 and $70 \mathrm{Mpc}$, respectively. Therefore the volumes of the cavities differ by a factor of 10 . A more extreme example is the MS0735.6+7421 cluster (McNamara et al. 2005) at redshift $z=0.22$, which has cavities with volume $\sim 10^{4}$ times larger than in NGC5813.

A systematic comparison of the AGN mechanical power and the gas cooling losses has been done for several dozens of objects (e.g., Rafferty et al. 2006; Hlavacek-Larrondo et al. 

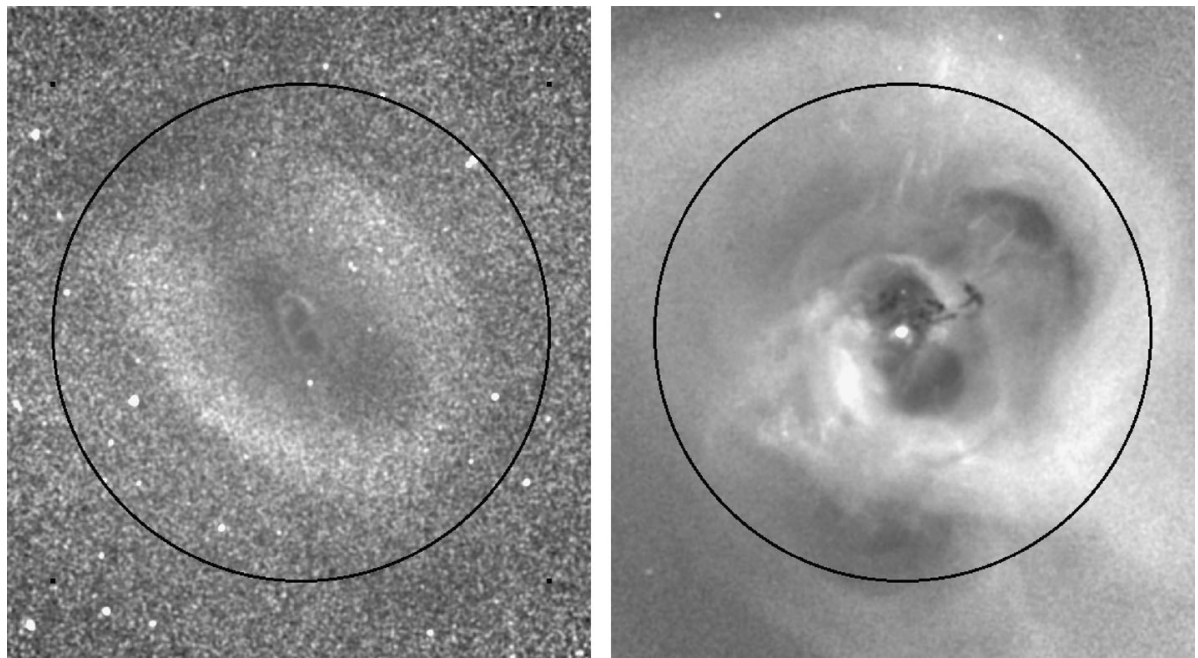

Fig. 6 Central $3^{\prime} \times 3^{\prime}$ region of the Chandra 0.6-2 keV images of NGC5813 (left) and the Perseus cluster (right). Cavities inflated by AGNs are clearly visible in both images. For NGC5813 two (or even three) generations of cavities are easily identifiable. The volume of the cavities in Perseus is a factor of $\sim 10$ larger than in NGC5813

Fig. 7 Comparison of the estimated AGN mechanical power and the ICM cooling losses for a sample of clusters. Adopted from

Hlavacek-Larrondo et al. (2012).

The correlation is evident, albeit with substantial scatter, suggesting that mechanical output is regulated to match the cooling losses

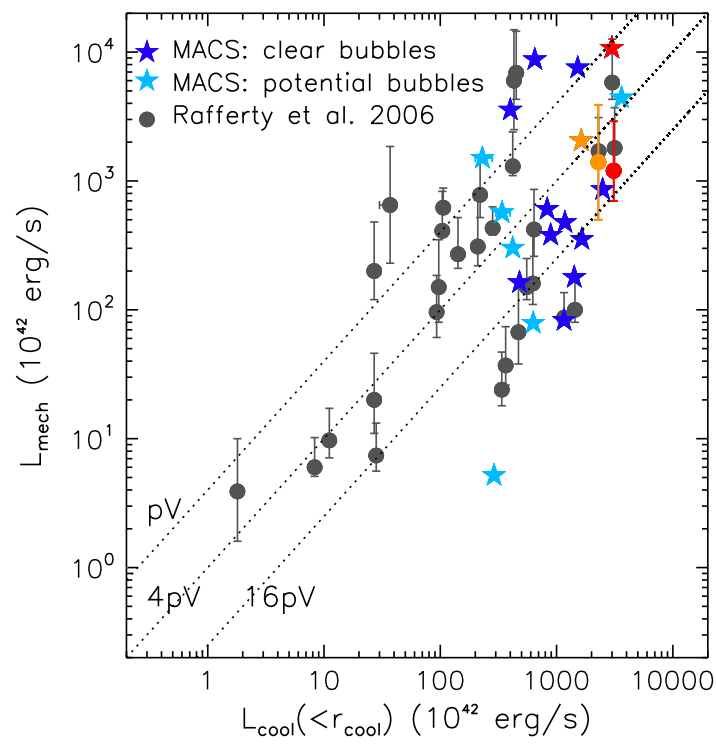

2012). These studies suggest an approximate balance between AGN energy input and cooling losses (see Fig. 7), implying that some mechanism is regulating the AGN power to maintain this balance. A natural way to establish such regulation is to link the accretion rate onto SMBHs with the thermodynamic parameters of the gas. Two scenarios are outlined below.

In the first scenario, known as "hot accretion", the classic Bondi formula (Bondi 1952) regulates the accretion rate of the hot gas onto the black hole and provides a link between the 
gas parameters and the mechanical power of an AGN (e.g., Churazov et al. 2002; Böhringer et al. 2002; Di Matteo et al. 2003).

The rate of spherically symmetric adiabatic gas accretion without angular momentum onto a point mass can be written as:

$$
\dot{M}=4 \pi \lambda(G M)^{2} c_{\mathrm{s}}^{-3} \rho \propto s^{-3 / 2},
$$

where $\lambda$ is a numerical coefficient, which depends on the gas adiabatic index $\gamma$ (for $\gamma=5 / 3$ the maximal valid value of $\lambda$ allowing steady spherically symmetric solution is $\lambda_{c}=0.25$ ), $G$ is the gravitational constant, $M$ is the mass of the black hole, $c_{\mathrm{s}}=\sqrt{\gamma \frac{k T}{\mu m_{\mathrm{p}}}}$ is the gas sound speed, $\rho$ is the mass density of the gas and $s=\frac{T}{n^{2 / 3}}$ is the entropy index of the gas.

Thus the Bondi accretion rate is proportional to $s^{-3 / 2}$ which increases when the gas entropy decreases. Assuming that the heating is directly proportional to the SMBH accretion rate (Heating $\left.=\epsilon c^{2} \dot{M}\right)$, there must be a value of gas entropy such, that heating balances cooling:

$$
s \approx 3.5\left(\frac{M}{10^{9} M_{\odot}}\right)^{4 / 3}\left(\frac{\epsilon}{0.1}\right)^{2 / 3}\left(\frac{L_{X}}{10^{43} \mathrm{erg} \mathrm{s}^{-1}}\right)^{-2 / 3} \mathrm{keV} \mathrm{cm}^{2}
$$

A lower/higher entropy than this value implies too much/little heating and therefore an overall increase/decrease of the accretion rate. Since in stable hydrostatic equilibrium the low entropy gas falls to the bottom of the potential well (the location of the SMBH), the energy input is controlled by the minimum value of the gas entropy in the whole central region. This provides a natural mechanism for self-regulation of the cooling and heating. Interestingly, in many nearby systems this simple prescription leads to an order of magnitude balance between cooling and heating (e.g., Churazov et al. 2002; Böhringer et al. 2002; Allen et al. 2006).

Another possibility is that some (sufficiently small) amount of gas is first able to cool from the hot phase down to low temperatures (e.g. down to $10^{4} \mathrm{~K}$ or below). Cold gas blobs then move in the potential well of the central galaxy, collide, lose angular momentum and feed the black hole (e.g., Pizzolato and Soker 2005; Gaspari et al. 2012). This model is known as a "cold accretion" scenario, as opposed to Bondi-type accretion straight from the "hot" phase. While these scenarios differ strongly in the physical process involved, they both advocate a negative feedback loop, when the SMBH affects the thermal state of the gas, which in turn affects the accretion onto the black hole.

\subsection{Link to Evolution of Galaxies}

A correlation of galaxy bulge properties and the mass of the SMBH (e.g., Ferrarese and Merritt 2000; Gebhardt et al. 2000) implies that the black hole and its parent galaxy affect each other. The clear evidence of mechanical feedback in nearby galaxy clusters suggests that the same mechanism may be relevant for the formation of massive ellipticals and for the growth of their SMBHs at $z \sim 2-3$. Three pre-requisites are needed for this scenario to work (i) a hot gaseous atmosphere in the galaxy is present, (ii) the black hole is sufficiently massive and (iii) large fraction of AGN energy is in mechanical form and the coupling of mechanical energy to the ICM is strong.

One can parametrize the magnitude of the feedback/gas heating $H$ with a simple expression:

$$
H\left(M_{\mathrm{BH}}, \dot{M}\right)=\left[\alpha_{M} \epsilon_{M}(\dot{m})+\alpha_{R} \epsilon_{R}(\dot{m})\right] 0.1 \dot{M} c^{2},
$$


where $\epsilon_{M}(\dot{m})$ and $\epsilon_{R}(\dot{m})$ characterize the efficiency of the transformation of accreted rest mass per unit time $\dot{M} c^{2}$ into mechanical energy and radiation respectively, while $\alpha_{M}$ and $\alpha_{R}$ are AGN-ICM coupling constants - the fraction of the released energy which is eventually transferred to the gas. The first pair of coefficients $\left(\epsilon_{M}(\dot{m}), \epsilon_{R}(\dot{m})\right)$ should come from accretion physics, while the second pair $\left(\alpha_{M}, \alpha_{R}\right)$ depends on the properties of the ICM and on the details of the AGN-ICM interaction. The value of $\alpha_{R}$ is typically very low $\alpha_{R} \lesssim 10^{-4}$ (Sazonov et al. 2004, 2005), while $\alpha_{M}$ can be close to unity (see above). This difference between $\alpha_{R}$ and $\alpha_{M}$ is the most important element of the mechanical feedback scenario.

Let us assume that the system ( $\mathrm{SMBH}+$ gaseous atmosphere) evolves to the state where the heating by the black hole is equal to the gas cooling losses (if such a state does exist). Thus the mass accretion rate is the solution of the equation:

$$
H\left(M_{\mathrm{BH}}, \dot{M}\right)=L_{\mathrm{cool}},
$$

provided $\dot{m}=\frac{\dot{M}}{\dot{M}_{\text {Edd }}} \leq 1$. In the opposite case, we set $\dot{M}=\dot{M}_{\text {Edd }}$. In other words, if the black hole cannot offset ISM cooling losses even at the Eddington rate then it will keep accreting gas at this rate. If there is a solution of Eq. (7) at $\dot{m} \leq 1$ then at this rate an equilibrium between heating and cooling is possible. We will see below that several distinct solutions of Eq. (7) are possible (some of them are unstable).

Let us now assume that the mechanical output $\epsilon_{M}(\dot{m})$ is large at low accretion rate, but decreases at high accretion rates (so-called "radio mode"). The radiative output $\epsilon_{R}(\dot{m})$ is on the contrary large at high accretion rates and decreases at low accretion rates. The mechanical and radiative outputs corresponding to this scenario are shown in Fig. 8 with the thin blue and red curves respectively. The above assumptions are motivated by X-ray and radio observations of several X-ray binaries (e.g., Gallo et al. 2003) and AGNs (e.g., Owen et al. M87) and can also be supported by theoretical arguments for radiatively inefficient accretion flows (e.g., Ichimaru 1977; Rees et al. 1982; Narayan and Yi 1994). For instance, the nucleus of M87 can be regarded as the prototypical example of an AGN in the low accretion rate mode, when kinetic power of radio emitting outflows exceeds its radiative power. At the same time, there are black holes in binary systems in the high accretion regime, which are very bright in X-rays, but show no evidence for a powerful outflow.

We now set $\alpha_{M} \sim 0.7$ and $\alpha_{R} \sim 10^{-4}$. The ICM heating by mechanical and radiative power (obtained by multiplying the curves by 0.7 and $10^{-4}$ ) is shown by the thick blue and red curves respectively. Finally the total ICM heating rate (the sum of two curves) is plotted as a thick black curve. Once the black hole is sufficiently massive, there are two possible solutions with the heating balancing ICM cooling losses (points marked as A and B in Fig. 8). The point $\mathrm{B}$ is certainly unstable since an increase of the mass accretion rate causes a decrease of the heating rate. The system will evolve from state B into one with lower or higher accretion rate. If the system goes into a high accretion rate mode then the feedback power drops and enhanced cooling boosts the accretion rate towards the Eddington value. Finally, the black hole mass reaches the level where AGN heating exceeds ICM cooling at any (sufficiently high) accretion rate. The gas entropy increases, the mass accretion rate drops and the system then switches to the stable state at low accretion rate, low radiative efficiency and high mechanical efficiency. This is the so called "radio-mode" which presumably describes M87 now. In the simple scenario outlined above the black hole at the center of the cooling core first looks like a QSO and then jumps to a state of a low luminosity AGN as shown with green line in Fig. 8.

The time evolution of the black hole mass, accretion rate and radiative luminosity is sketched in the right panel of Fig. 8. The black hole initially accretes at the Eddington rate 
Fig. 8 Top: illustration of gas heating and cooling in elliptical galaxies (Churazov et al. 2005). The thick solid line shows, as a function of the SMBH accretion rate, the heating rate due to outflow, which is complemented/dominated by radiative heating near the Eddington limit. Horizontal dashed lines show the gas cooling rate. The upper cooling line represents a young galaxy in which a large amount of gas is present and/or the black hole is small. Feedback from the black hole is not able to compensate for gas cooling losses and the black hole is in the QSO stage with a near-critical accretion rate, high radiative efficiency and weak feedback. As the black hole grows it moves down in this plot. The black solid dot marks the termination of this stage, when the black hole is first able to offset gas cooling, despite the low gas heating efficiency. The lower cooling line illustrates present day ellipticals: a stable solution exists at low accretion rates when mechanical feedback from the black hole compensates gas cooling losses. The radiative efficiency of accretion is very low and the black hole growth rate is very slow. Bottom: possible time evolution, corresponding to the figure above: the black hole accretes at the Eddington rate until its mass is large enough so that even weak feedback does not allow a stable solution. At later times the AGN switches into the low accretion rate mode, and the radiative efficiency drops dramatically
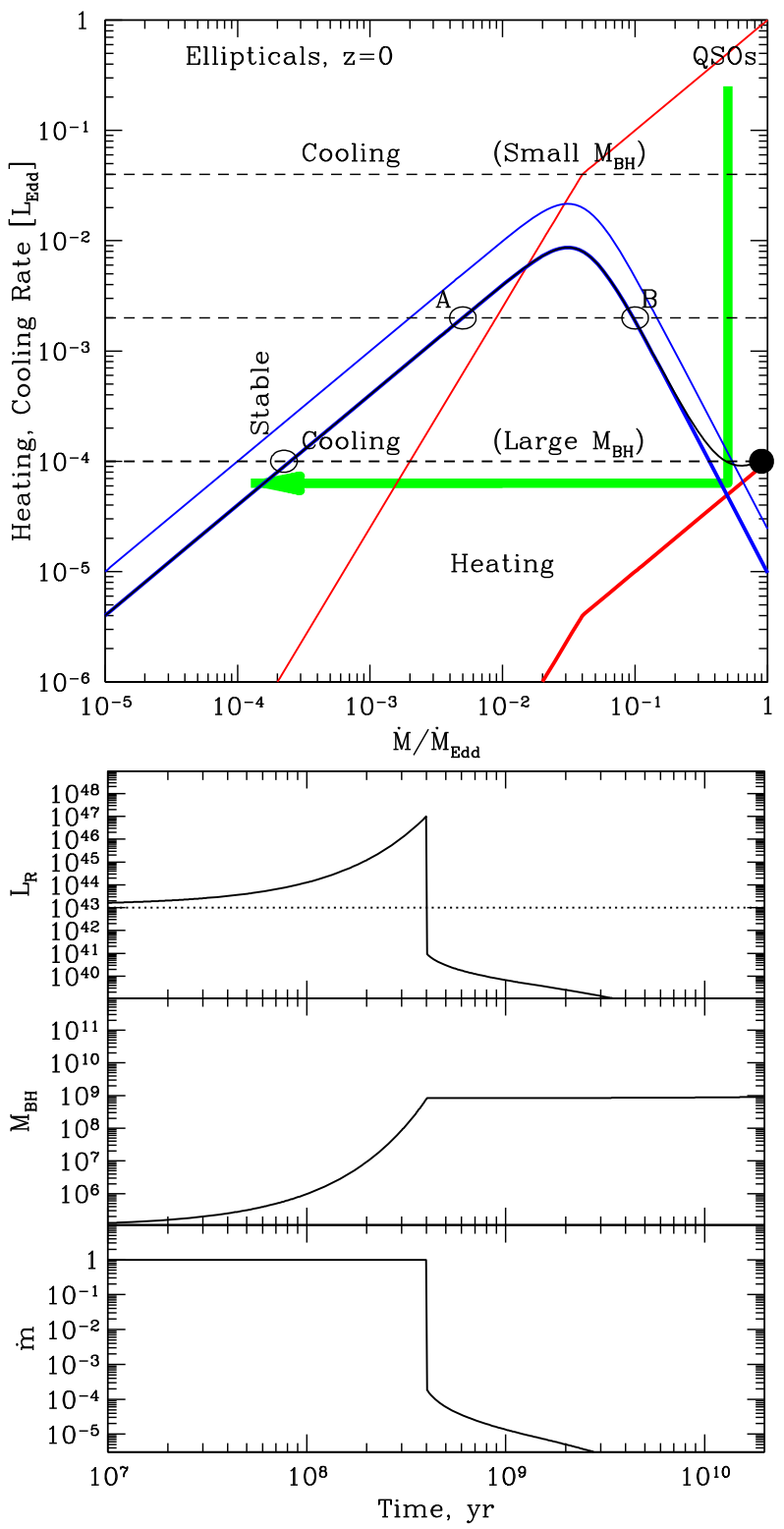

Time, yr

and grows exponentially with limited impact on the ICM. This fast growth and QSO-type behavior of the black hole continues until its mass is large enough so that even with pure radiative feedback, heating can offset cooling losses. After the system moves to the low accretion state the luminosity and the black hole growth rate drops by a large factor of order the ratio of the coupling constants $\alpha_{M} / \alpha_{R} \sim 10^{4}$ (or more if an ADAF-type scenario is adopted).

The above consideration is of course an overly simplified picture. Various prescriptions of the "radio-mode" feedback have been tested (e.g., Croton et al. 2006; Bower et al. 2006) 
in the semianalytic models coupled with the numerical simulations of structure formation of the Universe. In general, the key element of mechanical AGN feedback-the ability to provide good coupling of the AGN and the ICM-seems to be able to resolve the difficult issue of over-cooling and excess star formation in the most massive halos.

\subsection{Conclusions About AGN Feedback}

AGN Feedback in galaxy clusters is a rapidly developing area in astrophysics. It depends on a combination of various physical processes, ranging from physics of accretion to cosmological evolution of the most massive systems in our Universe. Much of the physics involved is still poorly understood. At the present epoch, AGN feedback prevents gas cooling in massive elliptical galaxies and clusters, but its role at higher redshifts is only beginning to emerge today (e.g. Hlavacek-Larrondo et al. 2012; McDonald et al. 2013). All this leaves much opportunity for future observational and theoretical studies.

\section{Galactic Feedback and Metal Enrichment in Clusters}

During the formation history of clusters of galaxies, metals have been continuously produced by the stars in the member galaxies. In the earliest epoch of star formation, about $500 \mathrm{Myr}$ after the 'Big bang', Population III stars formed from the primordial gas. The nature of this stellar population is still uncertain, but probably it consisted of intermediate and high-mass stars (Vangioni et al. 2011). The first metals produced by this population were mostly ejected into the surrounding medium, leading to an initial metal abundance of about $10^{-4}$ times Solar (Matteucci and Calura 2005). This relatively low metal abundance was enough to allow the gas to cool more efficiently through spectral line emission, resulting in an epoch of increased star formation, which peaked around a redshift of $z \sim 2-3$.

This peak of the Universal star formation rate roughly coincides with the build-up of a hot Intra-Cluster Medium in the massive galaxy clusters. Dilute metal-poor gas accreted from the cluster surroundings is mixed with the gas expelled by galactic winds driven by supernova explosions. A combination of compression, accretion shocks, supernova heating and AGN feedback boosted the ICM temperature to more than 1 million Kelvin. In such a hot environment, star formation in the cluster was effectively quenched (Gabor et al. 2010). While the build-up of the ICM continued, the star formation rate dropped to very low levels, which explains the old stellar populations observed in local galaxy clusters today.

Due to their deep gravitational potential wells, clusters have retained the metals ejected into their ICM. Metal abundances observed in the ICM of local clusters thus provide an integrated record of their enrichment history. The low star-formation rates in cluster galaxies since $z \sim 2-3$ form an interesting contrast to the enrichment history of our own galaxy, where the star-formation rate showed a smaller decline. Clusters of galaxies therefore provide a unique insight in the chemical enrichment due to stellar populations before $z \sim 2$.

\subsection{Sources of Metals}

Most of the elements heavier than beryllium are produced in supernovae. Some elements, like nitrogen and sodium, are ejected into the medium by Asymptotic Giant Branch (AGB) stars. There are two distinct types of supernova explosions, type Ia and core-collapse, that each have a separate role in metal enrichment. Core-collapse supernovae yield the bulk of 
Fig. 9 Expected abundances measured in a $120 \mathrm{ks}$

XMM-Newton observation of Sérsic 159-03 (bottom panel), which is a typical bright local cluster. The statistical error bars were obtained from de Plaa et al. (2006). The estimates for the SNIa, SNcc, and AGB contributions are based on a sample of 22 clusters de Plaa et al. (2007) and two elliptical galaxies (Grange et al. 2011). The top panels show the typical range in SNIa and IMF models with respect to the statistical error bars in the observation

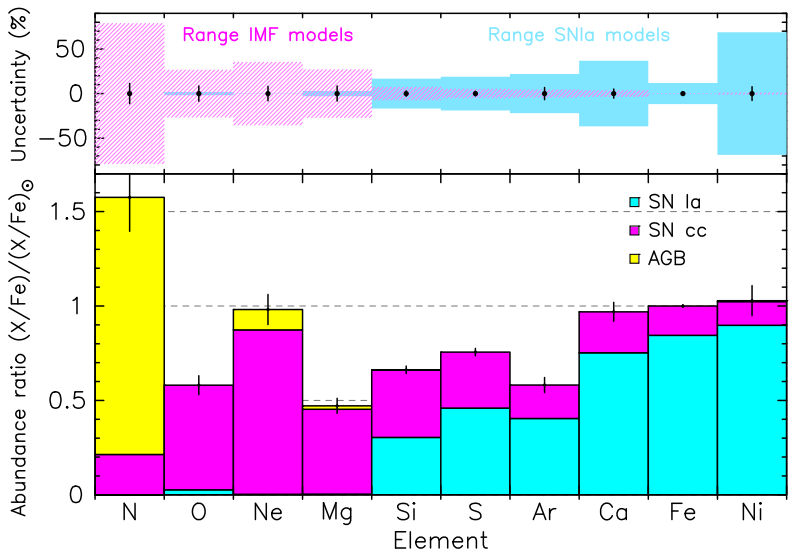

the elements in the mass range from oxygen to silicon, while type Ia supernovae produce mostly elements from silicon to nickel (see Fig. 9).

Supernova type Ia models, however, do not agree well on the exact yields for each element. Uncertainties on the yields of, for example, calcium and nickel are about 40-60\%. The underlying cause is the very interesting progenitor problem of type Ia supernovae. Recent optical observations of type Ia supernovae show variations in their spectral properties. So far, it has been challenging to link an observed type Ia supernova to a progenitor type (see e.g. Howell 2011). Two main progenitor scenarios are being considered. The first is the 'classical' type Ia, where a white dwarf accretes matter from a companion star in the Red Giant phase. If the accretion rate is right, the mass and temperature of the white dwarf can grow to a point where carbon fusion ignites. This is close to the Chandrasekhar limit of 1.4 solar masses. The carbon ignites explosively and unbinds the entire white dwarf. The second progenitor channel is a scenario where two white dwarfs merge. They spiral toward each other through the emission of gravitational radiation and the less massive star is accreted onto the more massive one, until carbon is ignited and the star explodes. It is clear that these progenitor channels allow a range of possible explosion scenarios and metal yields, which makes accurate predictions challenging.

The yields of core-collapse supernovae are currently better established. The main uncertainty in the core-collapse yield of an entire stellar population is the Initial-Mass Function (IMF). To obtain the total yield for a population, the yields calculated for individual masses need to be integrated over the IMF (Tsujimoto et al. 1995). Therefore, the observed abundances of metals in the oxygen to silicon mass range can constrain the IMF of the cluster's stellar population above $\sim 8$ solar masses.

Below $8 M_{\odot}$, intermediate-mass stars in their AGB phase are a source of nitrogen and sodium. Yields for these sources for several mass bins are available (Karakas 2010) and also need to be integrated over the IMF, like core-collapse yields, to obtain the yield for the whole stellar population. Measuring the abundances of AGB products therefore puts constraints the low-mass end of the IMF.

\subsection{Abundance Measurements in X-Rays}

The soft X-ray band between 0.1 and $10 \mathrm{keV}$ is very suitable for abundance studies because it in principle contains spectral lines from all elements between carbon and zinc. In clusters of galaxies, the hot plasma is in (or is very close to) collisional ionization equilibrium, 
Fig. 10 Abundance ratios fitted with supernova yields from the WDD2 SNIa model (Iwamoto et al. 1999) and a SNcc model with an initial metallicity $Z=0.02$ and a Salpeter IMF. The calcium abundance appears to be underestimated. It can not explain the $\mathrm{Ar} / \mathrm{Ca}$ ratio measured in this XMM-Newton sample of 22 clusters. (Adapted from de Plaa et al. 2007)

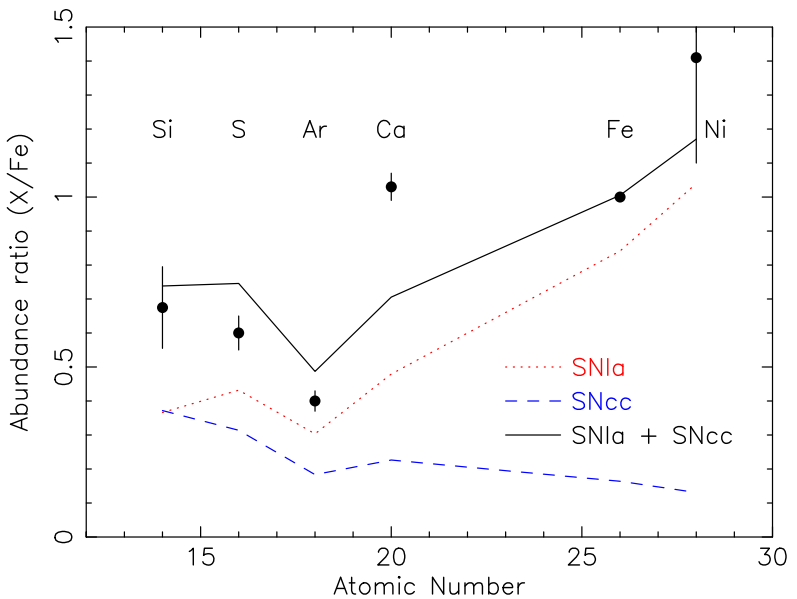

which makes it relatively easy to predict the emitted X-ray spectrum. The advance of X-ray spectroscopy has enabled the study of abundances in clusters and yielded interesting results (see e.g. Werner et al. 2008; de Plaa 2013, for more extended reviews on this topic).

The first attempt to link cluster abundances to supernova yields was done with the ASCA satellite (Mushotzky et al. 1996). With this instrument it was already possible to measure the abundances of $\mathrm{O}, \mathrm{Ne}, \mathrm{Mg}, \mathrm{Si}, \mathrm{S}, \mathrm{Ar}, \mathrm{Ca}, \mathrm{Fe}$ and Ni. From these, and other ASCA studies the picture emerged of an ICM that was enriched early in its formation history with corecollapse supernova products and later by type Ia supernovae.

With the launch of XMM-Newton, a telescope with a substantial effective area and spectral resolution (through the Reflection Grating Spectrometer, RGS) became available, which enabled deep abundance studies in larger cluster samples. In de de Plaa et al. (2007), for example, 22 clusters were analyzed and abundances were measured in their core regions. If the average abundances of the sample are compared to supernova type Ia models, the calcium abundance appeared to be systematically higher than expected by the models (see Fig. 10). Possible explanations for this high calcium abundance include an unexpected difference in the type Ia explosion mechanism or an increased importance of progenitor systems where helium is accreted on the white dwarf, because explosive He fusion is expected to yield more calcium. Although a systematic error in the determination of the $\mathrm{Ca}$ abundance cannot yet be fully excluded (detailed analysis did not show any problem), this measurement can constrain supernova type Ia models. Note that the fit shown in Fig. 10 includes Ca. If Ca is excluded a better fit for $\mathrm{S}$ and $\mathrm{Ar}$ is obtained.

If a combination of a type Ia model and core-collapse model fits, their ratio is an indication of the relative contribution of type Ia supernovae to the enrichment. Bulbul et al. (2012) developed an extention to the APEC model that is able to fit the type Ia to core-collapse ratio directly to the spectra. This ratio depends strongly on the used models (de Grandi and Molendi 2009). But despite of this uncertainty, multiple groups (e.g. Bulbul et al. 2012; Sato et al. 2007) report a type Ia contribution of 30-40\%, which is consistent with optical data.

In cool clusters and groups, the RGS spectrometer aboard XMM-Newton is able to measure carbon and nitrogen abundances. These elements are not produced in large quatities in supernovae, but appear to originate from metal-poor massive stars or AGB stars. The exact origin is still subject of debate (Romano et al. 2010). RGS observations by Werner et al. (2006a, 2006b) and Grange et al. (2011) have shown nitrogen to be very abundant around el- 
Fig. 11 Measured cluster abundances versus redshift. The top panel shows the abundances up to $0.6 r_{500}$ and the lower panel shows the abundances when the inner region up to $0.15 r_{500}$ is ignored. Adapted from Baldi et al. (2012)

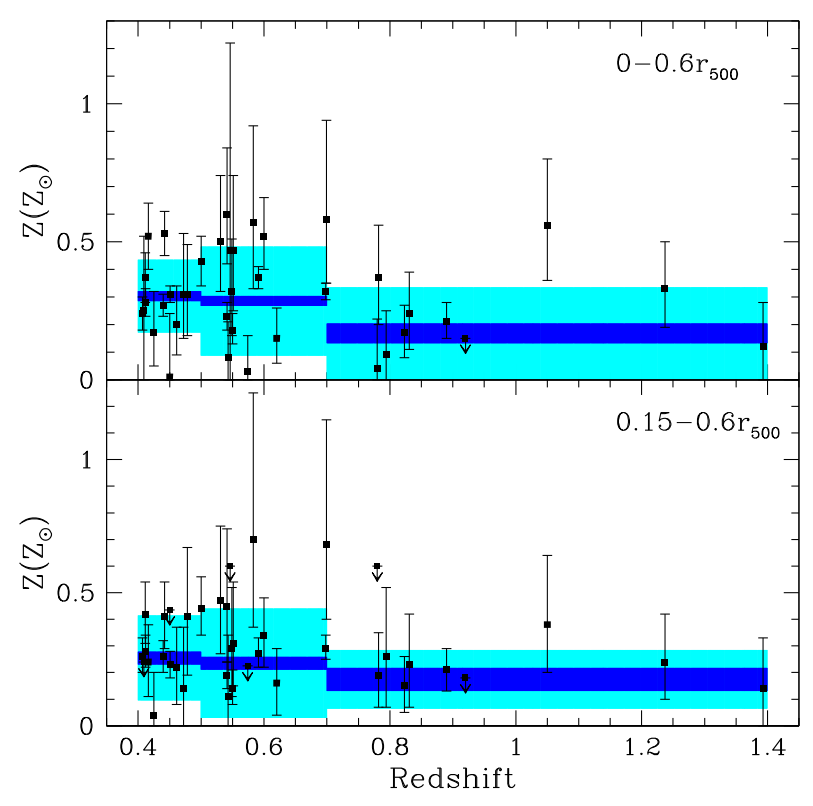

liptical galaxies. The high abundance of nitrogen cannot be explained by supernovae alone. A population of intermediate-mass AGB stars is therefore likely responsible for the high nitrogen content of the ICM.

With the Japanese Suzaku satellite, launched into low-earth orbit in 2005, abundance studies are performed in the outskirts of clusters (see Reiprich et al. 2013, for a recent and complete review of Suzaku interesting results). In these low surface-brightness areas, the low Suzaku background level is favorable above XMM-Newton. Recently, in an elaborate study of the outskirts of the Perseus cluster with Suzaku, Werner et al. (2013) found that the iron abundance distribution is surprisingly smooth in the outskirts. This points toward a scenario of an early enrichment of the hot X-ray gas with iron, well before $z \sim 2$. This result confirms earlier indications that chemical enrichment occured very early in the development of the universe and probably also before the formation of clusters.

One of these earlier indications was found in a study of a sample of high redshift clusters between $z=0.3-1.3$ by Baldi et al. (2012). Although the scatter in the measured metal abundance as a function of redshift is large, a significant trend in the metal abundance up to $z=1.3$ was not found (Fig. 11), indicating that the chemical enrichment mechanisms have not added a lot to the enrichment since $z=1$.3. Deeper observations of high redshift clusters would be necessary to build a large enough sample to confirm the lack of a metallicity trend with redshift.

\section{Magnetic Feedback Processes}

Galaxies play a key role in the enrichment of the ICM or IGM, not only as far as heavy elements are concerned, but also regarding the magnetization. According to the standard bottom-up scenario of lowest galactic masses, primeval galaxies must have injected much of their ISM into the IGM during the initial bursts of star formation, thereby "polluting" large volumes of intergalactic space because of their high number density. 
Kronberg et al. (1999) were the first to raise the question whether low-mass galaxies could have made a significant contribution to the magnetization of the IGM (apart from more massive starburst galaxies and AGN). Owing to their large number (observed and predicted in a CDM cosmology) and their injection of relativistic particles, they could have played a cardinal role in the context of this cosmologically important scenario. If true, it is to be expected that dwarf galaxies are "wrapped" in large envelopes of previously highly relativistic particles and magnetic fields, which are pushed out of them during epochs of vigorous star formation. Bertone et al. (2006) have discussed this more quantitatively and made predictions for the strengths of magnetic seed fields to be then amplified by large-scale dynamos over cosmic time. In particular, they also predict the existence of magnetic voids.

Donnert et al. (2009) and Dubois and Teyssier (2010) performed numerical models of supernova-driven winds in dwarf galaxies. Their simulations can provide an understanding of the origin of intergalactic magnetic fields at the level of $10^{-4} \mu \mathrm{G}$. Beck et al. (2013) presented a first numerical model of supernova-driven seeding of magnetic fields by protogalaxies.

The existence of winds in low-mass galaxies is inferred from the observed kinematics of the gas (measured with slit spectroscopy), but can arguably be also inferred from measurements of the temperature of the hot (X-ray-emitting) gas. For instance, Martin (1998) found the outflow velocities in NGC1569 to exceed the escape speed, and della Ceca et al. (1996) derived the temperature of its hot, X-ray-emitting gas to exceed the virial temperature. The transport of a relativistic plasma out of this galaxy is strongly suggested by radio continuum observations of dwarf galaxies. Kepley et al. (2010) studied the radio halo in NGC1569, which extends out to about $2 \mathrm{kpc}$ at $1.4 \mathrm{GHz}$. The dwarf irregular NGC4449 also possesses a low-frequency radio halo (Klein et al. 1996).

Of course, in magnetizing the ICM/IGM, low-mass galaxies have been competing with AGN (Rees 1987). Judging from the radio luminosities of the "culprits", it is clear that nevertheless low-mass galaxies may have contributed significantly. While a typical starburst dwarf galaxy emits a monochromatic radio luminosity of $P_{1.4 \mathrm{GHz}} \approx 10^{20.5} \mathrm{~W} \mathrm{~Hz}^{-1}$, radio galaxies in the FRI/II transition regime have luminosities of $P_{1.4 \mathrm{GHz}} \approx 10^{24.7} \mathrm{~W} \mathrm{~Hz}^{-1}$. Hence, the radio power produced by $\mathrm{AGN}$ is about $10^{4}$ times larger than that of dwarf galaxies. On the other hand, $\Lambda \mathrm{CDM}$ cosmology with bottom-up structure formation implies that dwarf galaxies must have been formed in huge numbers.

The role of massive black holes in the build-up of strong magnetic fields in galaxies was addressed by Chakrabarti et al. (1994). They pointed out that galactic winds or collimated jets were able to disperse such magnetic fields over large volumes of the host galaxies and beyond. Xu et al. (2010) presented magneto-hydrodynamical simulations in which they studied the evolution of magnetic fields ejected by an AGN shortly after the formation of a galaxy cluster. They showed that, if the magnetic fields are ejected before any major mergers occurring in the forming cluster, they can be spread throughout the cluster, with further subsequent amplification by turbulence in the ICM. It should be noted at this point that central so-called "mini-halos", which are bright extended radio sources located in the centers of cooling-flow clusters (e.g. Perseus A, Hydra A, Virgo A), cannot possibly magnetize large cluster volumes, as they are pressure-confined (e.g. de Gasperin et al. 2012).

\section{Cold Fronts in Galaxy Clusters}

Among the first results from high-resolution cluster images obtained with Chandra was the discovery of sharp edges in the X-ray surface brightness in merging clusters A2142 and 

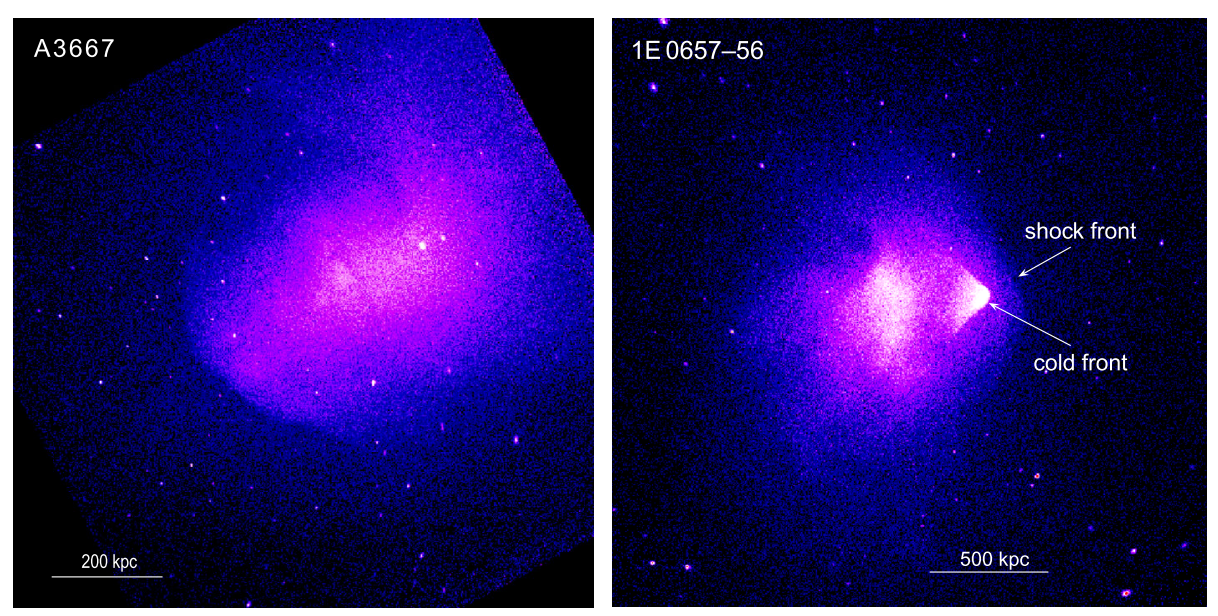

Fig. 12 Prominent cold fronts in the Chandra X-ray images of A3667 (MV07) and the Bullet cluster (Markevitch 2006). In the Bullet cluster, the surface of the cool "bullet" is a cold front; its supersonic motion generates a prominent bow shock

A3667 (Markevitch et al. 2000) (M00); (Vikhlinin et al. 2001a) (V01). Figure 12 shows deep Chandra images of A3667 and the Bullet cluster, both of which exhibit such brightness edges. The characteristic shape of the brightness profiles across these edges corresponds to a projected abrupt jump of the gas density at the boundary of a roughly spherical body (M00). The Bullet cluster shows two prominent edges, one at the nose of the dense cool "bullet" and another ahead of it. Their physical nature is revealed by radial profiles of the gas density, pressure and specific entropy, shown in Fig. 13. As one can guess already from the image, the outer edge is a bow shock - the dense side of the edge is hotter, with the pressure jump satisfying the Rankine-Hugoniot jump conditions (Markevitch 2006).

At the same time, the boundary of the bullet has a different physical nature - it separates two gas phases with very different specific entropies that are in approximate pressure equilibrium at the boundary. The denser side of this edge has a lower temperature, opposite to what's expected for a shock front, which is how the two phenomena can be distinguished observationally. The edge in A3667 and those in A2142 have the same sign of the temperature jump as that at the bullet boundary. These features in clusters they have been named "cold fronts" (V01). The term "contact discontinuity" is sometimes used, but it implies continuous pressure and velocity between the gas phases, whereas these structures in clusters may have discontinuous tangential velocity, as we will see below. Cold fronts turned out to be much more ubiquitous than shocks and have been observed with Chandra and XMM-Newton in many clusters and even galaxies (e.g., Machacek et al. 2005; see Markevitch and Vikhlinin 2007 (MV07), for a detailed review). If the dense gas cloud is moving with respect to the ambient gas, there will be a ram pressure component contributing to the pressure balance near the front (M00), which makes it possible to estimate the velocity of the cloud (V01).

Cluster cold fronts have two main physical causes, both related to mergers. The obvious one, originally proposed by M00 for the two fronts in A2142, involves an infalling subcluster ram-pressure stripped of its outer gas layers, which leaves a sharp boundary between the dense remnant of the subcluster's core and the less dense, hotter gas of the main cluster flowing around it. The bullet in the merging Bullet cluster and the NGC1404 galaxy falling 
Fig. 13 Schematic radial profiles of the gas density, pressure and specific entropy in a sector crossing the bullet and the bow shock in the Bullet cluster and centered on the bullet's center of curvature (see Fig. 12). The shock front and the bullet boundary have sharp density jumps of similar amplitudes; however, the shock at $r \approx 50^{\prime \prime}$ exhibits a large pressure jump and a slight entropy increase, while the bullet boundary at $r \approx 12^{\prime \prime}$ is in near pressure equilibrium but separates gases with very different entropies-it is a cold front (reproduced from MV07)

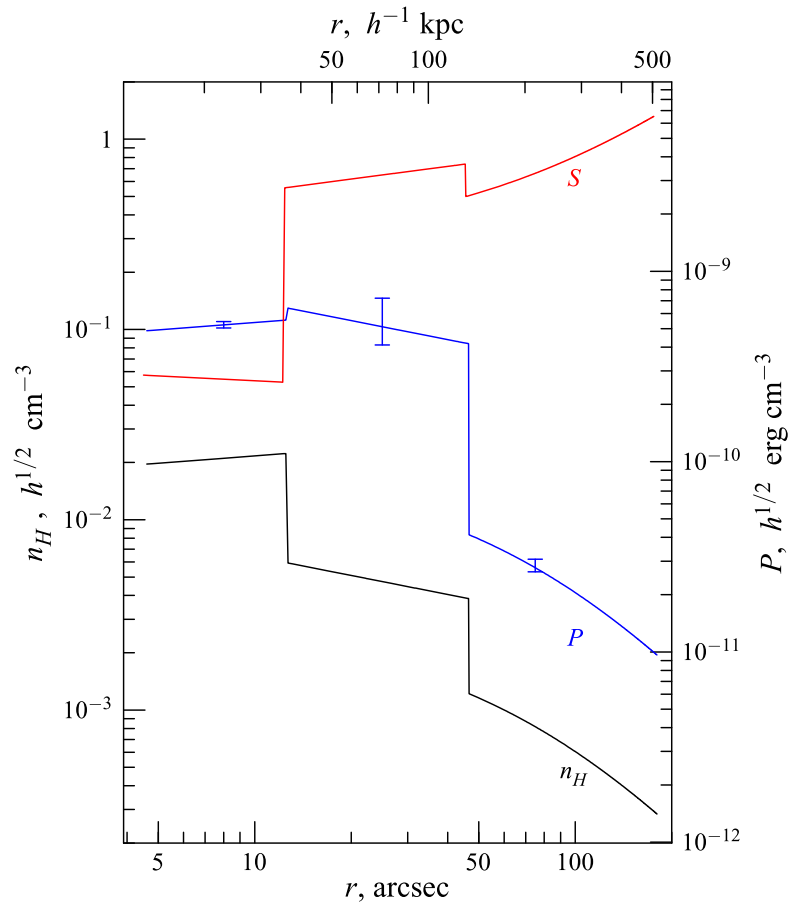

into the Fornax cluster (Machacek et al. 2005) appear to be such "stripping" fronts. ${ }^{1}$ However, cold fronts have also been observed in the cores of the majority of relaxed clusters that show no signs of recent merging (Mazzotta et al. 2001; Markevitch et al. 2001, M01; Markevitch et al. 2003; Ghizzardi et al. 2010). These fronts are typically more subtle in terms of the density jump than those in mergers, and occur close to the center $(r \lesssim 100 \mathrm{kpc})$, with their arcs usually curved around the central gas density peak. An example is seen in the Ophiuchus cluster (Fig. 14a). A detailed study of such a front in A1795 by M01 has shown that the gas on two sides of the front has different centripetal acceleration. This led those authors to propose that the dense gas of the cool core is "sloshing" around the center of the cluster gravitational potential, perhaps as a result of a disturbance of the potential by past mergers. While M01 envisioned radial "sloshing", Keshet et al. (2010) offered a more plausible scenario with a tangential flow of the cool gas beneath the fronts being responsible for centripetal acceleration.

Ascasibar and Markevitch (2006) (A06) have reproduced this phenomenon in highresolution hydrodynamic simulations of idealized binary mergers. To explain the absence of the gas disturbance on large scales, the small infalling subcluster should have no gas (only the collisionless dark matter-perhaps having lost its gas during previous stages of infall), thus disturbing only the gravitational potential of the main cluster without generating shocks in the gas. Such a disturbance results in a displacement between the gas peak and the collisionless dark matter peak, which sets off sloshing, which continues for several

\footnotetext{
${ }^{1}$ It appears that the original M00 scenario for A2142, which involved two surviving gas cores, is not correct (Tittley and Henriksen 2005; MV07). Instead, the two original fronts, the front discovered at a large radius (Rossetti et al. 2013), and yet another front seen close to the center in a deep Chandra observation-all concentric - are "sloshing" fronts discussed below. The "stripping" scenario does work in some other clusters.
} 

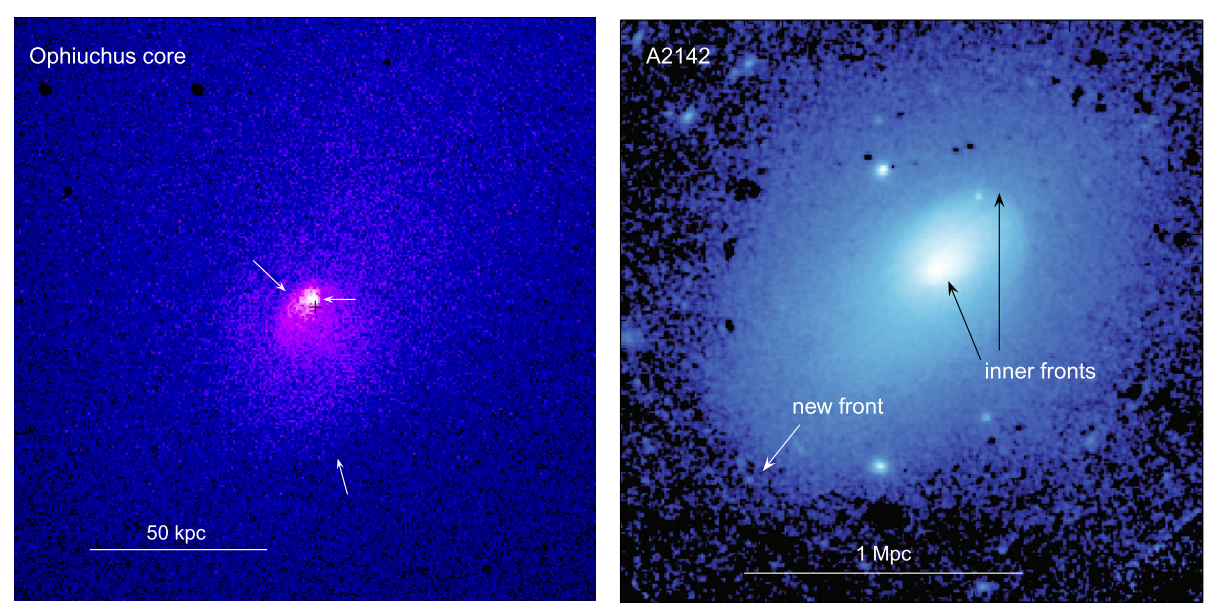

Fig. 14 Left: a series of "sloshing" cold fronts at different radii in the cool core of the Ophiuchus cluster, shown by arrows in this archival Chandra image. (The subtle outermost front is seen better in a more coarsely binned image.) Black cross marks the center of the cD galaxy. Right: a cold front discovered in A2142 at a distance of $1 \mathrm{Mpc}$ from the center (marked "new front") by Rossetti et al. (2013) in this XMM-Newton dataset. The original cold fronts in the central region (M00) are shown by black arrows

billion years, generating concentric cold fronts in a spiral pattern (if the merger had any angular momentum), as is often observed in cool cores. All that is required is a radial gradient of the specific entropy (which is always present in relaxed clusters) and an initial gas displacement. The idea that this class of cold fronts is the result of oscillations of the dark matter peak caused by a merger has been first proposed by Tittley and Henriksen (2005); the initial displacement may also be caused by the passage of a mild shock (Churazov et al. 2003; Fujita et al. 2004). Sloshing fronts are easily detected in cool cores, often delineating their boundary, but they are not confined to cool cores-in A06, a sufficiently strong initial disturbance caused detectable fronts to propagate to large radii. Indeed, cold fronts of this nature (that is, not associated with any infalling subclusters) have recently been found at $r \sim 0.7-1 \mathrm{Mpc}$ in Perseus (Simionescu et al. 2012) and in A2142 (Rossetti et al. 2013), see Fig. 14b. Note that this does not mean that the cluster gas oscillates from the center all the way out to those large distances. While sloshing does begin as a physical displacement of the gas density peak from the potential peak, the fronts propagate outwards as waves-see Fig. 8 in A06 and Nulsen and Roediger (2013), who describe sloshing and the resulting cold fronts as superposition of $\mathrm{g}$-mode oscillations in the stratified cluster atmosphere.

Core sloshing has a number of important effects on clusters. One is seen in the X-ray image of the Ophiuchus cluster (Fig. 14a; a matching snapshot from the numeric simulations can be seen in Fig. 7c of A06). The gas density peak (which contains the lowest-entropy gas of the cool core) is completely displaced from the center of the $\mathrm{cD}$ galaxy (shown by a cross). This would temporarily starve the central AGN of its fuel and may stop its activity. ZuHone et al. (2010) showed that sloshing can also facilitate heat transport from the hot reservoir outside the core into the cool core via mixing, which can compensate for most of radiative cooling in the core, provided that gas mixing is not suppressed. Keshet et al. (2010) and ZuHone et al. (2011) showed that tangential velocity shear in a sloshing core should amplify and reorder the initially tangled magnetic field. This effect has observable consequences for the shapes of cold fronts, which offers an independent tool to study those magnetic fields, as discussed below. There is also a physical connection between gas sloshing and cold fronts 
Fig. 15 X-Ray surface brightness profile across the cold front in A3667 (V01, MV07). Red line shows a best-fit model of a density jump that is smoothed with $\sigma=11 \mathrm{kpc}$, which is the m.f.p. for Coulomb diffusion from the dense side to the less dense side of the front. If diffusion were present, the front would have been smeared by several times this width; such diffusion is clearly excluded by the data

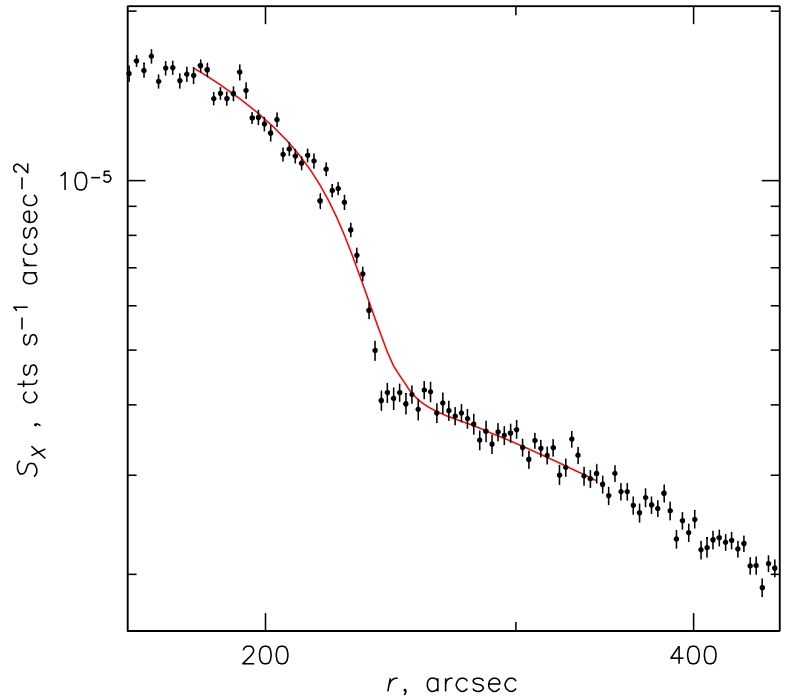

on one side and the radio-emitting ultrarelativistic electrons in the cluster cores on the other (Giacintucci et al. 2014; ZuHone et al. 2013a).

\subsection{Physics of Cold Fronts}

Cold fronts can be used to study the microphysics of the intracluster plasma. One property of the cold fronts observed with Chandra that is immediately striking is their sharpness. Figure 15 shows an X-ray brightness profile across the prominent front in A3667 (Fig. 12a). Vikhlinin et al. (2001a) pointed out that the front is sharper than the mean free path for Coulomb collisions. Indeed, red line in Fig. 15 shows a best-fit model that includes broadening of the front with a width equal to the Coulomb m.f.p. for diffusion from the cool inner side to the hot outer side of the front. Such broadening is clearly inconsistent with the data, which means the diffusion across the front is suppressed. Ettori and Fabian (2000) pointed out that the existence of the observed temperature jumps at cold fronts implies that thermal conduction is also strongly suppressed. Vikhlinin et al. (2001b) proposed (within the "subcluster stripping" scheme of M00) that the motion of the infalling subcluster through a tangled magnetic field frozen into the ambient gas of the main cluster would naturally form an insulating layer of the field oriented strictly along the front surface, as a result of the field "draping" around an obstacle, as originally proposed by Alfvén to explain the comet tails. This effect is shown in a simulation by Asai et al. (2005) in Fig. 16. As pointed out by Lyutikov (2006), such draping can amplify the field in the narrow layer immediately outside the front to values approaching equipartition with thermal pressure (compared to magnetic pressures of order $1 \%$ of thermal pressure in the rest of the cluster). Such a layer would completely suppress diffusion and thermal conduction across the front, while the magnetic tension of such a layer may stabilize the front against Kelvin-Helmholtz instability (Vikhlinin et al. 2001b; MV07), which we will see below in a simulation.

If a cluster with a cool core initially has a tangled magnetic field, when the core is disturbed and starts sloshing, the field is rapidly stretched by the tangential gas velocity shear (ZuHone et al. 2011), forming layers of amplified field oriented along the surface of the cold front form. These layers are located under the front surfaces, unlike for the draping effect 


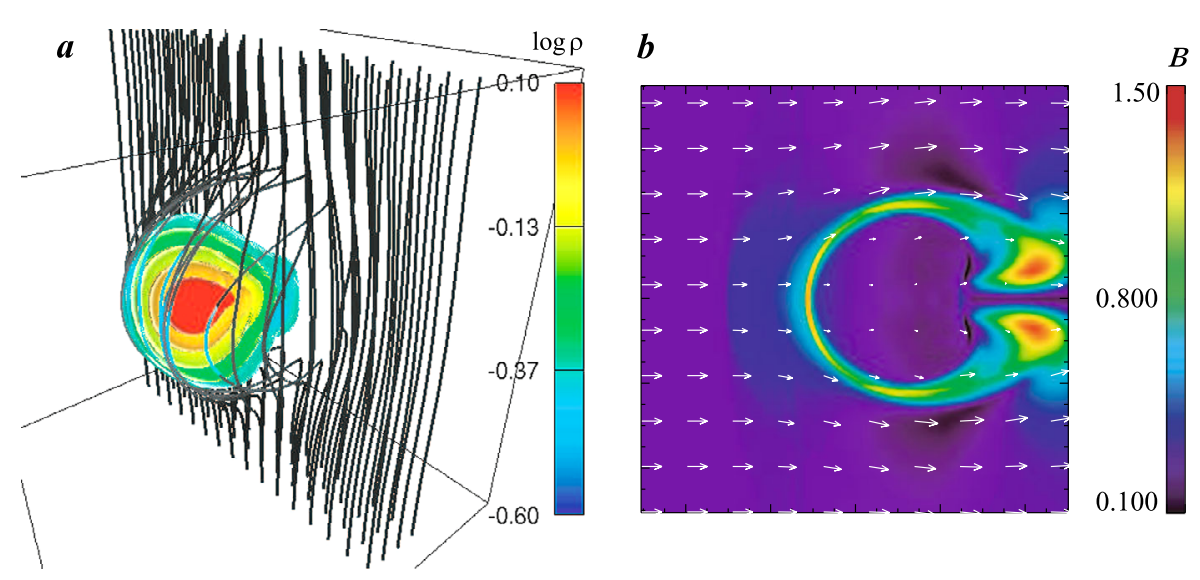

Fig. 16 Magnetic field draping around an infalling subcluster (panel a). This results in a layer of amplified and ordered field immediately outside the cold front (panel b). The field is initially uniform, but the effect for a tangled field is similar. (Reproduced from Asai et al. 2005)
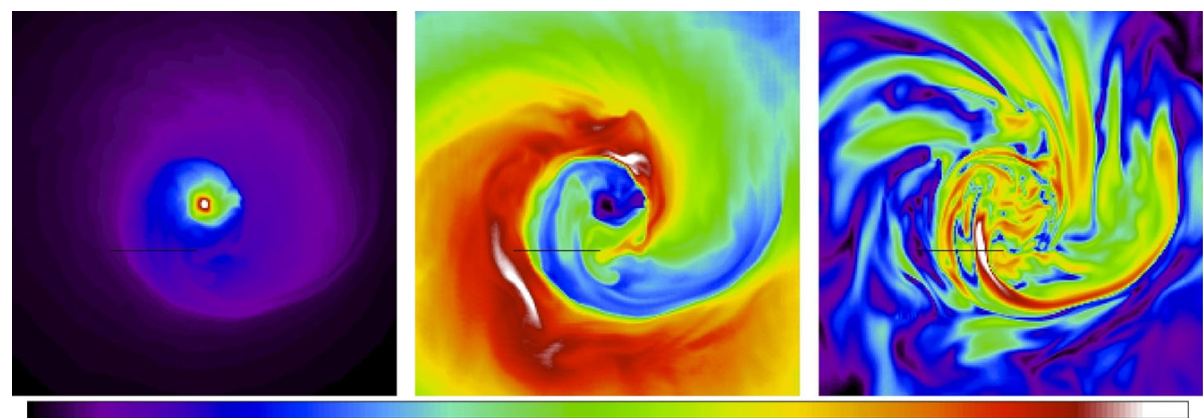

Fig. 17 The magnetic field around a "sloshing" cold front. Left panel shows X-ray surface brightness, middle panel shows gas temperature, and right panel shows the magnetic field strength. Unlike for the "stripping" front (Fig. 16), the field is strongly amplified inside the front. (Reproduced from ZuHone et al. 2011)

in a "stripping" front. A snapshot from the simulations of this process is shown in Fig. 17. Such layers can suppress KH instability of the front surface (Fig. 18) similarly to a draping layer. However, there is an important difference for the effective thermal conduction across the front.

If a subcluster falls into a cluster from a large distance, it will be thermally insulated from the ambient gas by a magnetic draping layer for as long as the subcluster survives as a coherent structure, because the initially disjoint magnetic field structures of the cluster and subcluster cannot connect at any stage of the infall (as long as there is no magnetic reconnection) - even when the gases are geometrically separated only by a thin cold front. Since heat is conducted only along the field lines, it is not surprising to see a cool infalling subcluster survive the immersion into the hot gas of the bigger cluster (e.g., the group falling into A2142 discovered by Eckert et al. 2014). However, for a sloshing cold front, thermal conductivity across the front may not be completely suppressed by the magnetic layer, as shown by simulations of ZuHone et al. (2013b). The reason is that prior to the onset of sloshing, the regions inside and outside the cluster core are connected by the field lines, 

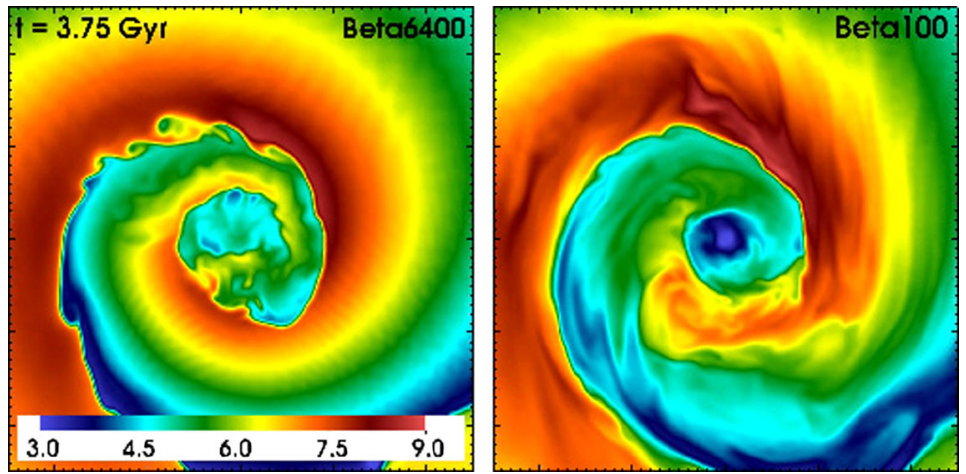

Fig. 18 The stabilizing effect of the magnetic field on cold fronts. Panel size is $500 \mathrm{kpc}$; color shows gas temperature (the scale is in $\mathrm{keV}$ ). Left panel shows a simulation with a weak field (initial plasma $\beta=6400$ ), right panel shows a stronger, more realistic field $(\beta=100)$. The realistic field suppresses $\mathrm{K}-\mathrm{H}$ instabilities, leaving relatively undisturbed cold fronts, similar to those observed. (Reproduced from ZuHone et al. 2011)

and while sloshing stretches most of them tangentially, it does not sever the connection completely. Those authors suggested that the existence of the temperature jumps across the sloshing cold fronts may therefore be used to constrain heat conduction along the field lines.

Another interesting (and completely unknown) plasma property that may be constrained by the observations of cold fronts is viscosity (MV07). Figure 19 (from ZuHone et al. 2015) shows a simulated sloshing core with the plasma viscosity modeled in different ways. The viscosity, either isotropic or anisotropic (it is likely to be the latter in the presence of magnetic fields), acts to suppress the KH instabilities, and the effect should be observable. Of course, the effect of viscosity is superimposed on the stabilizing effect of the magnetic layers discussed above, so the observations will most likely be able to constrain some combination of the two. Based on simulations without the magnetic field, Roediger et al. (2013) concluded that the effective isotropic viscosity should be significantly suppressed to explain the disturbed appearance of a cold front in Virgo.

This area of research is currently under rapid development, with high-quality observations of cold fronts being obtained and tailored numeric simulations being constructed, so interesting constraints on the microphysical properties of the cluster plasma can be expected soon.

\section{Shock Fronts and Non-thermal Components}

\subsection{Shock Fronts}

Shock waves in clusters of galaxies are the main agents to convert the kinetic energy of supersonic and superalfvénic plasma flows produced by gas accretion, merging substructures and AGN outflows into both the thermal and the non-thermal components. The shocks are essential to heat the gas and to produce the observed thermal and non-thermal radiation. The shock structures in plasma and their ability to create the non-thermal componentsenergetic charged particles and electromagnetic fields-depends on whether the shock is collisional or collisionless. The mean free path of a proton due to the Coulomb collisions is $\lambda_{\mathrm{p}} \approx 7 \times 10^{21} v_{8}^{4} n_{-3}^{-1}$ (here $v_{8}$ is proton velocity in thousands $\mathrm{km} \mathrm{s}^{-1}$ and $n_{-3}$ is the ambient gas number density in $10^{-3} \mathrm{~cm}^{-3}$ ). Note that an ion of charge $Z$ and atomic weight $A$ have a 


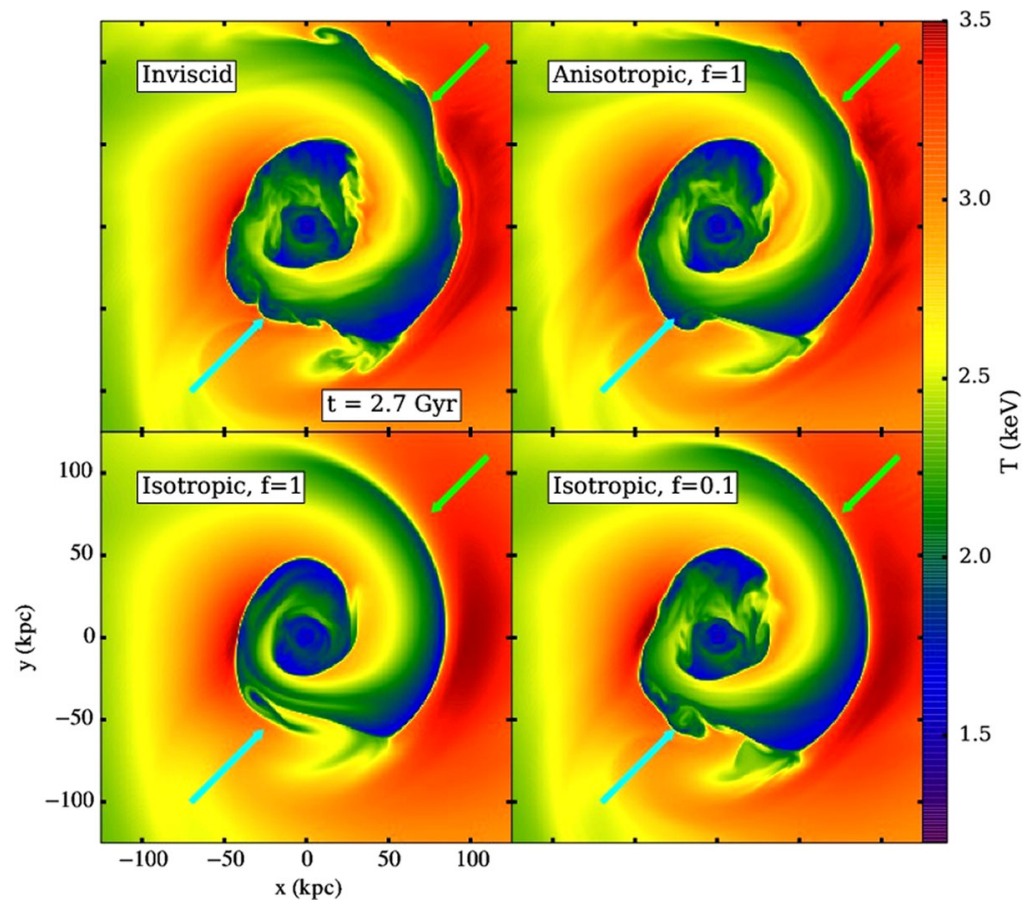

Fig. 19 Simulation of a sloshing core with different physical models for plasma viscosity: inviscid (with a weak magnetic field present), isotropic Spitzer viscosity, anisotropic Braginskii viscosity along the field lines, and isotropic viscosity suppressed by factor $f=0.1$. A highly suppressed viscosity is required to produce visible K-H eddies at cold fronts. (Reproduced from ZuHone et al. 2015)

gyroradius $r_{\mathrm{gi}}=3.3 \times 10^{9}\left(A T_{\mathrm{keV}}\right)^{1 / 2}\left(Z B_{\mu \mathrm{G}}\right)^{-1} \mathrm{~cm}$ in a magnetic field $B_{\mu \mathrm{G}}$ measured in $\mu \mathrm{G}$. The Coulomb mean free path is much larger than the proton gyroradius (as well as the ion inertial length which we shall introduce later) at all particle energies of interest in the cluster and thus the plasma shocks in clusters are expected to be collisionless as it is the case in the hot interstellar and interplanetary plasmas.

A specific feature of the collisionless shocks is the mechanism of the flow momentum and the energy dissipation by means of the excitation and damping of collective electromagnetic fluctuations providing numerous degrees of freedom with a very broad range of the relaxation times and spatial scales. Moreover, the collisionless shock may accelerate particles to ultra-relativistic energies resulting in a broad particle energy spectra formation. The multi-scale nature of the shock with a strong coupling between the scales makes the problem of laboratory studies and theoretical modeling of the structure of the collisionless shock to be very complicated. However, some basic features of the collisionless shock physics were established from direct interplanetary plasma observations, imaging and spectroscopy of shocks in supernova remnants and computer simulations (see e.g. the review by Treumann 2009).

The shock flow dissipation mechanisms depend on the flow velocity, magnetization and shock obliquity. Shocks of low enough Mach numbers $\mathcal{M}_{\mathrm{s}}<\mathcal{M}_{\text {crit }}$ are able to dissipate the kinetic energy of the flow by anomalous Joule dissipation. In the lack of Coulomb collisions the dissipation is usually associated with the anomalous resistivity provided by wave-particle interactions. Such shocks are called subcritical and $\mathcal{M}_{\text {crit }}$ is dubbed the first critical Mach 


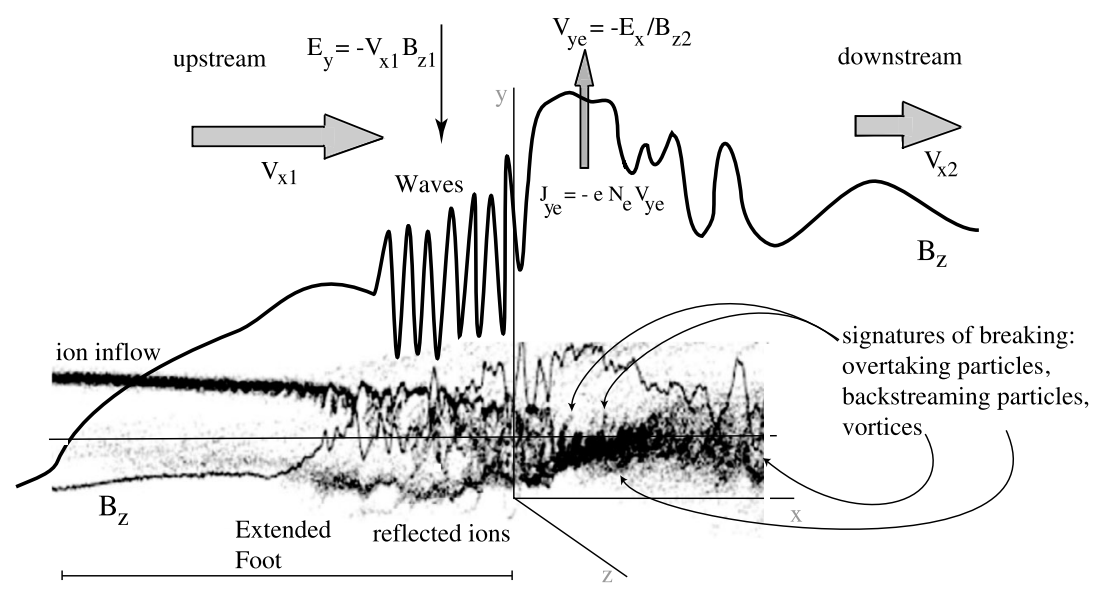

Fig. 20 Schematic structure of a perpendicular supercritical shock. The profiles of the magnetic field and plasma density at the shock transition region dominated by the ion reflection dissipation mechanism are shown. Charge separation over an ion gyro-radius in the shock ramp magnetic field produce the electric field along the shock normal which may reflect the incoming ions back upstream. The electric field along the shock front produced by conductive plasma motion in the magnetic field may accelerate particles by the electric drift acceleration mechanism. The magnetic field of the current carried by the accelerated back-streaming ions causes the magnetic foot in front of the shock ramp. Reproduced from Treumann (2009)

number (see e.g. Kennel et al. 1985). The structure of the shock transition in this case may be smooth and laminar, but this mainly occurs for low $\beta$ shocks. The magnetization parameter $\beta=8 \pi n T / B^{2}=\mathcal{M}_{\mathrm{a}}^{2} / \mathcal{M}_{\mathrm{s}}^{2} \approx 40 n_{-3} T_{\mathrm{keV}} B_{\mu \mathrm{G}}^{-2}$, characterizes the ratio of the thermal and magnetic pressures. The first critical Mach number is maximal for a quasi-transverse shock in the plasma with low magnetization $\beta>1$ and it is below 2.76. At large $\beta$ typical for the hot intracluster plasmas some transverse shocks as well as very weak shocks can be subcritical, while most of the shocks are supercritical. The width of the viscous transition in the collisionless subcritical shock wave can be estimated as $l_{\mathrm{e}} / \sqrt{\mathcal{M}_{\mathrm{s}}-1}$, where $l_{\mathrm{e}}=c / \omega_{\mathrm{pe}} \approx 1.7 \times 10^{7} n_{-3}^{-0.5} \mathrm{~cm}$. Here $\omega_{\mathrm{pe}}$ is the electron plasma frequency.

If the shock Mach number exceeds $\mathcal{M}_{\text {crit }}$ the anomalous resistivity is unable to provide the required dissipation rate to satisfy the Rankine-Hugoniot conservation laws at the shock. Then the shock became supercritical and its structure is turbulent. The dissipation mechanism of a supercritical shock is usually dominated by ion reflection. Some fraction of the incoming ions are reflected by a force which is a combination of electrostatic and magnetic fields and the reflected ion behavior depends on angle between the local upstream magnetic field and the local shock normal. In Fig. 20, taken from Treumann (2009), we illustrate the basic processes in the collisionless perpendicular shocks.

The width of the supercritical shock transition in case of a quasi-parallel shock may reach a few hundreds ion inertial lengths which is defined as $l_{\mathrm{i}}=c / \omega_{\mathrm{pi}} \approx 7.2 \times 10^{8} n_{-3}^{-0.5} \mathrm{~cm}$. Here $\omega_{\text {pi }}$ is the ion plasma frequency and $n$ is the ionized ambient gas number density measured in $\mathrm{cm}^{-3}$. The shock transition structure is unsteady in the case of magnetized shocks being a subject of shock front reformations which however are expected to be suppressed in the hot intercluster plasmas. The widths of viscous transition in the collisionless shocks is orders of magnitude less the Coulomb mean free path and it is below the spatial resolution of optical and X-ray observations even for a few kpc distance galactic supernova shocks.

Non-thermal energetic electrons are responsible for most of the observed radio emission from clusters, but the exact nature of particle acceleration process and the origin of the ob- 
served $\sim \mu \mathrm{G}$ strength magnetic fields in clusters are still under debate. From the energetic ground shock waves are the most natural accelerating agent in hot weakly magnetized plasmas. As we have learned from multi-wavelength observations of strong shocks in young supernova remnants in order for diffusive shock acceleration (DSA) to be fast enough to reach $100 \mathrm{TeV}$ regime particle energies observed in the sources, significant non-adiabatic magnetic field amplification is required (see e.g. Bell 2004; Amato and Blasi 2009; Bykov et al. 2012; Schure et al. 2012). The cosmic ray driven instabilities may provide a source of free energy for strong magnetic field amplification transferring a few percent of ram pressure of a strong shock (with $\mathcal{M}_{\mathrm{s}} \gg 1$ ) into fluctuating magnetic fields (see Fig. 11 in Bykov et al. 2014). This is indeed enough to provide $\mu \mathrm{G}$-magnetic fields behind the strong large scale accretion shock. The observed $\mu \mathrm{G}$ magnetic field strength is well above the field amplitude produced by the adiabatic compression of the intergalactic field at an accretion shock. Therefore it is possibly produced by CR driven instabilities at the strong accretion shock. The field amplification mechanism driven by instabilities of the anisotropic CR distribution produced by DSA is efficient if CRs get a substantial fraction of the shock ram pressure, which is expected in strong shocks with hard CR spectra. In the case of strong amplification of the fluctuating magnetic field in the shock precursor, the direction of the local magnetic field just before the viscous plasma shock transition will vary with time providing periods of both quasi-perpendicular and quasi-parallel configurations. This may differ from the case of weak shocks with steep spectra of accelerated particles which may not contain enough free energy in the high energy end of particle spectra to provide strong long-wavelength turbulence in the shock upstream.

Because of much higher pre-shock densities, the internal shocks with modest Mach numbers $\mathcal{M}_{\mathrm{s}}<4$ in clusters, which are propagating in a very hot intracluster plasma, dissipate more energy than the strong accretion shocks. Internal shocks with $2<\mathcal{M}_{\mathrm{s}}<4$ were shown in simulations by Ryu et al. (2003) to produce about a half of the total kinetic energy dissipation, while the internal shocks as a whole are responsible in this model for about $95 \%$ of gas thermalization. Microscopic simulations of electron acceleration in quasi-perpendicular shocks of $\mathcal{M}_{\mathrm{s}}<5$ were performed by Guo et al. (2014a). This particle-in-cell plasma modeling demonstrated that the repeated cycles of shock drift acceleration (SDA) may form power-law electron energy spectra. In a particular run with a quasi-perpendicular pre-shock magnetic field and $\mathcal{M}_{\mathrm{s}}=3$ they found that about $15 \%$ of the electrons were accelerated and a power-law electron energy spectrum with a slope of $q \approx 2.4$ was formed. The energy density carried by the accelerated energetic electrons in this simulation was about $10 \%$ of the bulk kinetic energy density of the incoming ions. This electron acceleration efficiency is much higher than that estimated from supernova remnant observations. The transverse box size in this simulation was about one ion gyro-radius. It is important to confirm the interesting result with larger simulation boxes since the shock structure is determined by ions and the model have to fulfill the requirements of the theory of charged particle motion in an electromagnetic field with one ignorable spatial coordinate by Jones et al. (1998).

The very long (Mpc scale) highly polarized radio structure observed in the merging galaxy cluster CIZA J2242.8+5301 by van Weeren et al. (2010) can be understood in this way, as the associated weak shock may not disturb the initial inter-cluster magnetic field in the shock upstream. The observed polarization favors a transverse shock configuration which may provide efficient shock drift acceleration of relativistic electrons (Guo et al. 2014a,b). This would require a very uniform magnetic field in the shock upstream. The radio structures and the X-ray emission of CIZA J2242.8+5301 cluster illustrating the location and the extension of the large scale shocks of moderate strengths are shown in Fig. 21 from Akamatsu et al. (2014). 
Fig. 21 Smoothed 0.5-2.0 keV band X-ray image (red) and WSRT $1.4 \mathrm{GHz}$ image of CIZA J2242.8+5301 (cyan). The thin yellow lines depict the approximate locations of the shock fronts confirmed by Suzaku. The figure is courtesy Akamatsu et al. (2014)

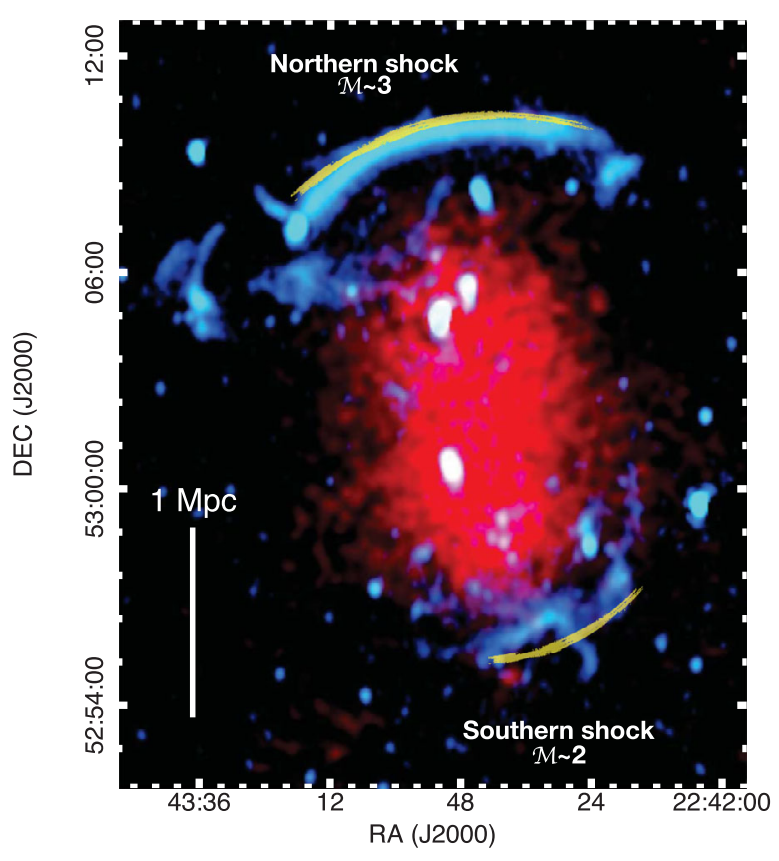

\subsection{Intracluster Cosmic Rays}

Both electrons and ions are likely accelerated at the shock fronts produced by mergers or supersonic outflows, but their subsequent evolution differ markedly. Since the ultra relativistic electrons have a radiative lifetime much shorter than the age of the cluster, they rapidly radiate most of their energy away and then may comprise a long lived population at Lorentz factors of around 100 where both Coulomb and radiation losses are longer than $10^{9}$ years (Petrosian et al. 2008). On the other hand, ions (protons mostly) lose only a small fraction of their energy during the lifetime of the cluster, and their diffusion time out of the cluster is even larger, so that they are stored in clusters for a cosmological time scale (Völk et al. 1996; Berezinsky et al. 1997).

The long lived non-thermal particle populations may be the subject of a consequent reacceleration by multiple shocks of different strengths as it is likely the case in galactic superbubbles and starburst regions (cf. Bykov 2001, 2014). Relativistic electrons can be re-accelerated in-situ by a long-wavelength MHD turbulence (Bykov and Toptygin 1993) which may be generated in the ICM during cluster mergers (Brunetti and Lazarian 2011; Brunetti and Jones 2014). The energy contained in both populations depends on the energy spectra of particles. Electrons of energies at about $50 \mathrm{MeV}$ are difficult to constrain from the observational point of view. Gamma-ray observations are used to constrain the energy density in relativistic particles (see e.g. Ackermann et al. 2014). Recently, Prokhorov and Churazov (2014) analyzed Fermi-LAT photons above $10 \mathrm{GeV}$ collected from the stacked 55 clusters selected from a sample of the X-ray brightest clusters. They obtained an upper limit of the pressure of relativistic protons to be $1.5 \%$ relative to the gas thermal energy density, provided that the spectral index $q$ of relativistic proton power-law distribution is 2.1, while for $q=2.4$ the limit is already about $6 \%$. These estimations assume that relativistic and thermal components are mixed. Similar results were reported by Ackermann et al. (2014) who analyzed another set of clusters at the photon energies starting from $500 \mathrm{MeV}$. 
The observations reported above have placed stringent limits on the pressure contained in the high-energy particle populations with hard spectra, while the constraints on the nonthermal components with steep enough spectra to be dominated by the sub-GeV particles remain to be established.

The most direct evidence of a non-thermal component within the intracluster medium comes from radio observations, that have revealed the presence of two main classes of Mpcscale diffuse synchrotron sources, generally called "radio halos" and "radio relics" (see e.g. Ferrari et al. 2008, 2012). The origin of cosmic rays in radio halos is still elusive, even though their existence has been known for decades. The morphological, spectral and polarization properties of the radio halos were attributed by Brunetti et al. (2001) to radiation of relativistic electrons accelerated by ICM turbulence. Alternatively, the radiating leptons could be of the secondary origin i.e. a product of hadronic collisions of (long-lived) nonthermal energetic protons with thermal nuclei (Dennison 1980; Blasi and Colafrancesco 1999). In this case, one should also expect $\gamma$-ray emission, which has not been seen so far. MHD simulations carried out by Donnert et al. (2010) indicated some problems in explaining the observed steepening of the synchrotron spectra of halos. However, hadronic models with energy-dependent CR proton transport coefficients may be able to reproduce the steep spectra (Enßlin et al. 2011).

Radio relics are considered to be related to DSA, even if some of current observational results suggest the need to review or refine electron acceleration models within this class of sources (van Weeren et al. 2012; Ogrean et al. 2014). Radio observations of most kinds of relic sources in clusters of galaxies show rather steep synchrotron spectra $\left(S_{v} \propto v^{-\alpha}\right)$, with $\alpha \gtrsim 1$ (see e.g. Miley 1980; Ferrari et al. 2008, 2012). As expected in DSA models, the spectral index $\alpha$ can change within the source, with a steepening from the front towards the back of the shock (see e.g. the spectacular radio relic in the cluster CIZA J2242.8, whose spectral index is ranging from 0.7 to about 1.7; Stroe et al. 2013). The corresponding indices of the relativistic lepton power-law spectra are $q \gtrsim 2.5$. The soft particle spectra are expected to be produced by relatively weak shocks in both the diffusive shock acceleration model (see e.g. Brüggen et al. 2012) and in the shock drift electron acceleration model by Guo et al. (2014a). The simulated statistical distribution of the merging shock strengths (see e.g. Ryu et al. 2003) peaks at rather weak shocks, and therefore the constrains on the pressure in relativistic leptons of Lorentz factors of about 100 and the non-thermal sub- and semirelativistic protons of soft energy spectra still need further substantiation.

Apart from radio and gamma-ray observations discussed above fine X-ray spectroscopy has a potential to study non-thermal components. To illustrate the effects of such a suprathermal electron distribution on data, Kaastra et al. (2009) simulated two X-ray microcalorimeter spectra extracted from a circular region with a radius of $1^{\prime}$ centered on the core of a bright cluster with a $0.3-10 \mathrm{keV}$ luminosity of $6.3 \times 10^{37} \mathrm{~W}$ within the extraction region, at an assumed redshift of $z=0.055$. In the simulation of the spectrum of a deep $100 \mathrm{ks}$ $\mathrm{X}$-ray micro-calorimeter observation, they assumed a post-shock downstream electron distribution for a Mach number of $\mathcal{M}=2.2$ and pre-shock temperature of $k T=8.62 \mathrm{keV}$ (i.e. $10^{8} \mathrm{~K}$ ) calculated with a kinetic model of electron heating/acceleration by a collisionless shock developed by Bykov and Uvarov (1999). In Fig. 22 taken from Kaastra et al. (2009) we present the 6.9-7.0 keV part of the spectrum (rest-frame energies) which shows the Fe XXVI Ly $\alpha$ lines and the Fe XXV j-satellite line simulated for a 100 ks observation with the X-ray micro-calorimeter aboard the ASTRO-H mission and also for that proposed for the future Athena mission. Enhanced equivalent widths of satellite lines are shown to be a good indicators of non-thermal electrons. The satellite line in the simulated spectrum is clearly stronger than that predicted by the thermal model with a Maxwellian electron distribution. The energy density in the supra-thermal electrons modeled by Guo et al. (2014a) 
Fig. 22 X-ray micro-calorimeter spectra from a $1^{\prime}$ region at the center of a bright cluster with a mixture of the thermal and supra-thermal electrons as simulated by Kaastra et al. (2009). Simulations made for 100 ks observations with Athena (top panel) and ASTRO-H (bottom panel). The energy distribution of electrons accelerated at $\mathcal{M}=2.2 \mathrm{Mach}$ number shock was calculated with the kinetic electron acceleration model by Bykov and Uvarov (1999). Crosses are simulated data points. Solid line: best-fit model to a pure Maxwellian plasma, with temperature $16.99 \mathrm{keV}$. Note the excess emission of satellite lines in the data, in particular the Fe XXVI-line
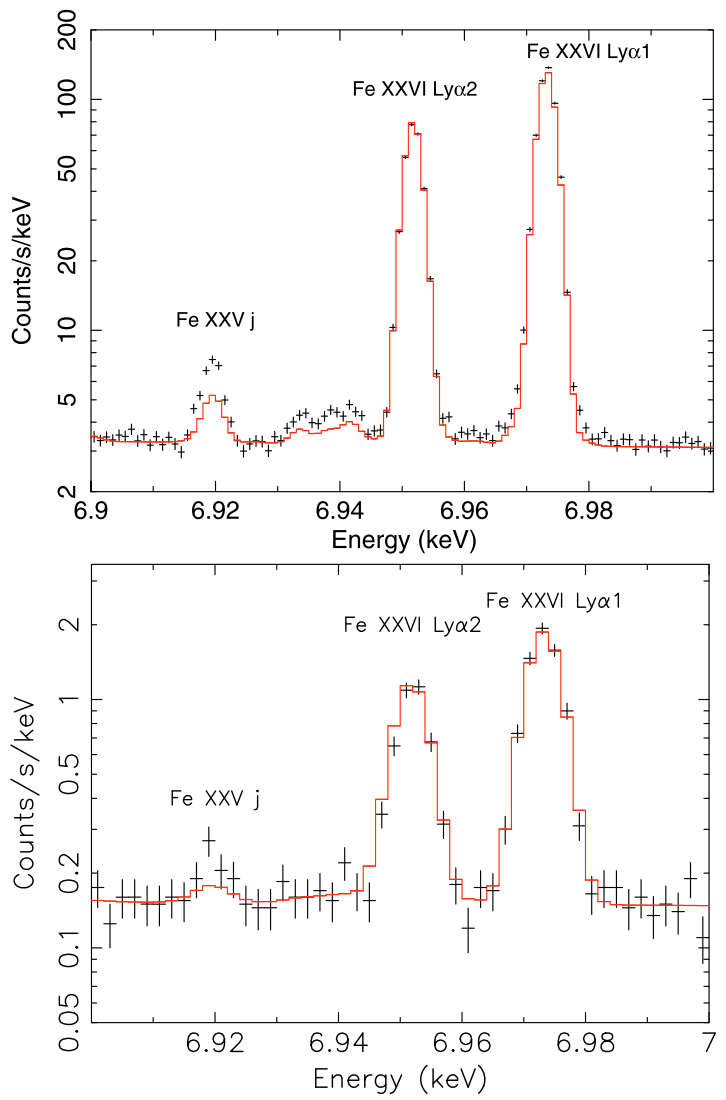

is larger than that was used in the simulations by Kaastra et al. (2009) and therefore it can be observationally tested in this way. This illustrates a good potential of high-resolution spectra obtained by future satellites with a large effective area to observationally reveal nonMaxwellian tails in the electron distributions.

\subsection{Intracluster Magnetic Fields}

The study of the linearly polarized emission from radio images is of cardinal importance in order to constrain the properties of intra-cluster magnetic fields.

Vacca et al. (2010) studied the power spectrum of the magnetic field associated with the giant radio halo in the galaxy cluster A665. They performed sensitive observations of this cluster with the Very Large Array at $1.4 \mathrm{GHz}$ and compared these with simulations of random three-dimensional turbulent magnetic fields, trying at the same time to reproduce the observed radio continuum emission from the halo. They constrained the strength and structure of the intracluster magnetic field by assuming that it follows a Kolmogorov powerlaw spectrum and that it is in local equipartition with relativistic particles. A central magnetic field strength of about $1.3 \mu \mathrm{G}$ was inferred for A665. The azimuthally averaged brightness profile of its radio emission suggests the energy density of the magnetic field to follow the thermal gas density, leading to an averaged magnetic field strength over the central $1 \mathrm{Mpc}^{3}$ of about $0.75 \mu \mathrm{G}$. An outer scale of the magnetic field power spectrum of $\sim 450 \mathrm{kpc}$ was estimated from the observed brightness fluctuations of the radio halo. 
Bonafede et al. (2011) used the Northern VLA Sky Survey to analyze the fractional polarization of radio sources out to 10 core radii from the centers of 39 massive galaxy clusters with the aim to find out how different magnetic field strengths affect the observed polarized emission along different sight lines through the clusters. They found the fractional polarization to increase towards the cluster peripheries, and their findings are in accord with a magnetic-field strength of a few $\mu \mathrm{G}$ in the centers. A statistical test gives no hint at any differences in depolarization for clusters with and without radio halos, but indicates significant differences of the depolarization of sources seen through clusters with and without cool cores.

Govoni et al. (2013) investigated the potential of new-generation radio telescopes (e.g. the Square Kilometer Array, SKA and its pathfinders) in detecting the polarized emission of radio halos in galaxy clusters. To this end, they used magneto-hydrodynamical simulations conducted by Xu et al. (2011, 2012), Beck et al. (2013) to predict the expected polarized emission of radio halos at $1.4 \mathrm{GHz}$. The synthetic maps of radio polarization were compared with the detection limits on polarized emission from radio halos set by current and upcoming radio interferometers. They show that both the angular resolution and sensitivity expected in future sky surveys at $1.4 \mathrm{GHz}$ using the SKA precursors and pathfinders (like APERTIF, ASKAP, Meerkat) are rather promising for the detection of the polarized emission of the most powerful $\left(L_{1.4 \mathrm{GHz}}>10^{25} \mathrm{~W} \mathrm{~Hz}\right)$ radio halos. Furthermore, the upgraded JVLA has the potential to detect polarized emission from strong radio halos already now, though with relatively low angular resolution. However, the detection of polarization signal in faint radio halos $\left(L_{1.4 \mathrm{GHz}}<10^{25} \mathrm{~W} \mathrm{~Hz}\right)$ has to await the fully deployed SKA.

\section{Turbulence in Clusters}

Turbulence and density fluctuations affect the basic assumptions behind hydrostatic equilibrium and hence the estimates of important cosmological parameters such as cluster masses. For this reason, but even more because of the interesting astrophysical processes involved, we discuss in more detail the various observational signatures of turbulence and density inhomogeneities.

We consider here constraints from measurements in the radio band and pressure maps, surface brightness distributions, resonant scattering and line broadening in the X-ray range.

\subsection{X-Ray View: Pressure and Surface Brightness Mapping}

Large scale plasma motions and turbulence in clusters primarily concerns fluctuations in the velocity field. However, they also lead to density and pressure fluctuations that can be measured with X-ray telescopes with sufficient spatial resolution and only modest spectral resolution (to measure temperatures).

Schuecker et al. (2004) described this method in a seminal paper on the Coma cluster. From a mosaic of XMM-Newton observations of Coma, spatial scales between $20 \mathrm{kpc}$ and 2.8 Mpc could be sampled. Their maps show-superimposed on the normal radial density and pressure gradients-fluctuations up to $2 \%$ in temperature and $5 \%$ in intensity. These fluctuations are correlated and agree with the expected adiabatic fluctuations of the pressure. The spatial power spectrum of these pressure fluctuations is in agreement with a Kolmogorov-like spectrum. They found that between 10-25\% of the total intracluster pressure is in the form of turbulence. 
More recently, Churazov et al. (2012) extended this work by also including higher spatial resolution observations of Coma with Chandra. Contrary to Schuecker et al. (2004), they consider density fluctuations. They find that the relative density fluctuations in Coma have amplitudes of 5 and $10 \%$ at scales of about 30 and $300 \mathrm{kpc}$, respectively. They also consider several other explanations for the observed density fluctuations. For instance, at large scales, gravitational perturbations due to the large $\mathrm{cD}$ galaxies and entropy variations due to infalling cold gas may produce apparent density fluctuations.

\subsubsection{Surface Brightness Profiles}

In the cluster outskirts, models predict that the X-ray emission should become more clumpy due to the shallow gravitational potential and the ongoing strong cluster evolution associated, for instance, with infall of galaxies or groups towards the growing mass concentrations. One may define a cluster clumping factor $C$ as

$$
C^{2}=\left\langle n^{2}\right\rangle /\langle n\rangle^{2}
$$

with $n$ the gas density and the brackets denoting averaging over volume.

Simionescu et al. (2011) used Suzaku observations of the outskirts of the Perseus cluster to determine the cluster properties around the virial radius and beyond. They find an apparent baryon fraction higher than the cosmic value at these large radii, which they reconcile by assuming a very clumpy medium at these distances.

Interestingly, Eckert et al. (2013), by combining pressure measurements from Planck with density profiles obtained from ROSAT, found a much lower clumping factor $C$ of about 1.2 at $R_{200}$, compared to $3-4$ by Simionescu et al. (2011). These differences may be associated to the precise way of averaging used in determining $C$.

\subsubsection{Direct Measurements of Line Broadening}

The most direct way to measure turbulence in clusters is to measure the spectral line broadening caused by the turbulence. Motion of the line-emitting ions will produce Doppler shifts. However, also thermal motion of the ions produces Doppler shifts. The width $\sigma_{T}$ for thermal motion of the ions (for a Gaussian velocity profile $\sim \exp \left(-\Delta v^{2} / 2 \sigma_{T}^{2}\right)$ ) is given by

$$
\sigma_{T} / c=0.00103 T_{\text {ion }} / \sqrt{M}_{\text {ion }},
$$

where the ion temperature $T_{\text {ion }}$ is expressed in $\mathrm{keV}$ and the ion mass $M_{\text {ion }}$ in atomic units. Thus, for hydrogen and an ion temperature of $1 \mathrm{keV}$ the thermal broadening is larger than the turbulent broadening if the turbulent velocity dispersion in the line of sight $\sigma_{\mathrm{v}}$ is less than $300 \mathrm{~km} \mathrm{~s}^{-1}$. For the more relevant case of iron (atomic mass 56.25) the thermal velocity dispersion is always less than $200 \mathrm{~km} \mathrm{~s}^{-1}$ (Fig. 23), and hence turbulence is relatively easily detected provided that the detector has sufficient energy resolution to measure the line broadening.

At this moment the only instrument capable of measuring line broadening in clusters is the RGS reflection grating spectrometer on board of XMM-Newton. However, because this is a slit-less dispersive instrument, the spatial extent of a cluster gives an additional line broadening effect, and this must be modeled properly. Only some cool core clusters have sufficiently small size to allow for such a study. In addition, there is some uncertainty in the calibration of the instrumental line spread function. With these caveats in mind, Sanders 
Fig. 23 Thermal velocity dispersion in the line of sight corresponding to the thermal motion of emitting ions, for oxygen silicon and iron ions, as a function of gas temperature

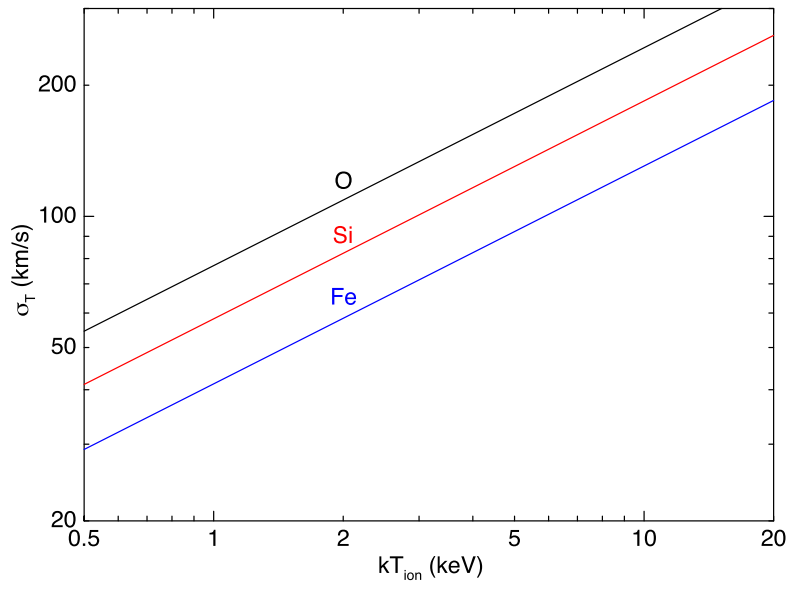

et al. (2011) studied a sample of 62 clusters, groups and individual ellipticals. They found upper limits for the turbulent velocity of $200 \mathrm{~km} \mathrm{~s}^{-1}$ for five of their studied cases, and one case (Klemola 44) with a measured broadening of $1500 \mathrm{~km} \mathrm{~s}^{-1}$. Optical observations show this latter cluster to be a very disturbed system. For the sample as a whole, 15 sources appear to have less than $20 \%$ of their thermal energy in the form of turbulence.

Clearly, better measurements are needed. ASTRO-H (expected launch 2015) will contain a calorimeter with about $6 \mathrm{eV}$ spectral resolution $\left(300 \mathrm{~km} \mathrm{~s}^{-1}\right.$ at the iron lines), that does not suffer from the spatial blurring inherent to the RGS. Turbulent broadening of spectral lines is expected to be measurable down to $50-100 \mathrm{~km} \mathrm{~s}^{-1}$, ultimately limited by the calibration of the instrument. Measuring cluster turbulence is one of the key scientific questions that this satellite will address.

While ASTRO-H has sufficient spectral resolution to measure the turbulence, its spatial resolution of the order of an arcmin will limit the amount of mapping that is possible. In the longer future, the proposed ESA mission Athena (launch 2028) will have a significantly higher spatial resolution, in addition to a spectral resolution of a few $\mathrm{eV}$ and a much higher effective area. This will allow to study turbulence also for a significant number of clusters at much higher redshifts. In addition, the spectral resolution will be sufficient to study line profiles in detail (e.g. Inogamov and Sunyaev 2003).

\subsubsection{Resonance Scattering}

Resonance scattering offers a unique tool to study turbulence in clusters. The hot plasma in clusters of galaxies is in general optically thin, which means that all radiation that it produces can freely escape. However, a few of the strongest resonance lines have an optical depth of the order of unity. Photons emitted in such lines can be scattered several times before they finally escape. For clusters of galaxies, the method was pioneered by Gilfanov et al. (1987), see Churazov et al. (2010) for a review. Essentially, line photons from resonance lines emitted from the center of the cluster towards the observer will be scattered out of the line of sight, but are not lost. This leads to an apparent dimming of the line towards the cluster center, but a brightening towards the outer parts. Because of the higher surface brightness near the cluster center, this dimming of resonance lines towards the center is the easiest to observe. 
Fig. 24 Simulated Astro-H spectra from the core (0.5-1.5 arcmin annulus) of the Perseus Cluster for $100 \mathrm{ks}$ observation. Points show the simulated spectra with and without resonant scattering for different levels of turbulence, parametrized through the effective Mach number $\mathcal{M}$. Note that $100 \mathrm{ks}$ observation is sufficient to detect the resonant scattering signal even if gas motions are present. (Adapted from Zhuravleva et al. 2013)

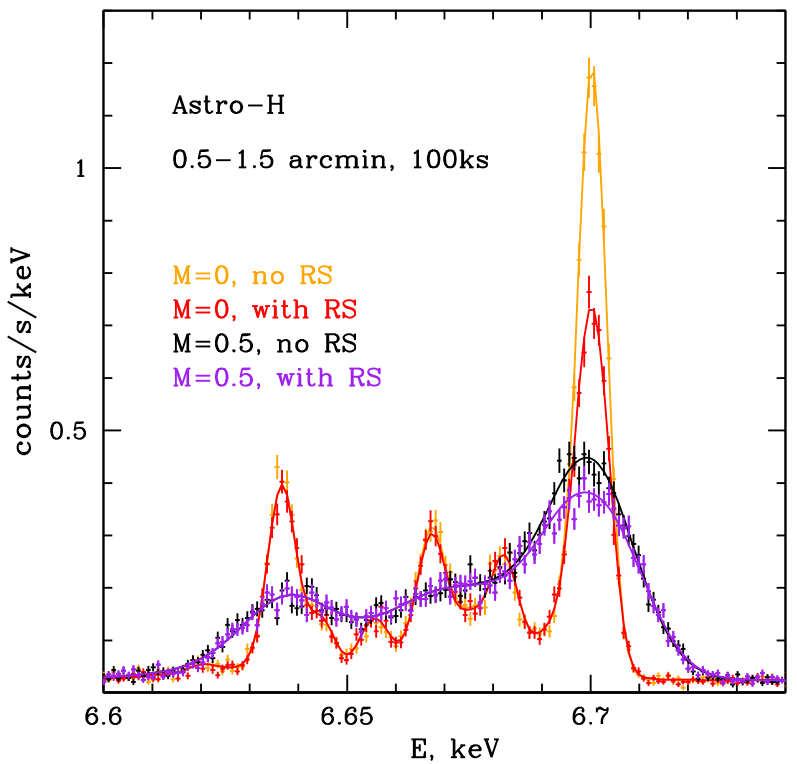

To make fully use of this effect, two lines of the same ion are needed, with different oscillator strength. Then from the radial profile of their line ratio (combined with information about the temperature and density profile of the cluster obtained from spectral modeling), the optical depth of the scattering line can be determined. This optical depth depends on the ratio of the turbulent to thermal energy density in the gas. Therefore this method directly probes the turbulence. Since the core of the cluster is typically much brighter than the outskirts, the optical depth is particularly sensitive to the radial component of the velocity field and small-scale eddies, while large-scale coherent motions tend to shift the line energy, but do not affect the optical depth (Zhuravleva et al. 2011).

One of the most promising ions to apply this method is Fe XXV. The $1 \mathrm{~s}-2 \mathrm{p}$ and $1 \mathrm{~s}-3 \mathrm{p}$ transitions of this ions have high and low oscillator strength, respectively. While BeppoSAX and ASCA observations of the Perseus cluster indicated the possible presence of a strong scattering effect and hence low levels of turbulence (Molendi et al. 1998; Akimoto et al. 1999), subsequent observations with XMM-Newton showed that the Fe XXV 1s - 3p line is contaminated with nickel line emission; correcting for that results in insignificant resonance scattering hence the presence of substantial turbulence, with characteristic speeds of 0.36 times the sound speed and hence a turbulent pressure of about $10 \%$ of the thermal pressure (Gastaldello and Molendi 2004; Churazov et al. 2004). Future ASTRO-H mission will have sufficient sensitivity and energy resolution to unambiguously identify signatures of the resonant scattering in the Fe XXV triplet (Fig. 24) in a 100 ks observations.

Another interesting diagnostic ion is Fe XVII. This has a strong resonance line at $15 \AA$, and by comparing this line with other Fe XVII lines, Xu et al. (2002) discovered resonance scattering in the elliptical NGC4636 using RGS data. This work was extended by Werner et al. (2009) using five giant elliptical halos, and using in addition to the RGS spectra also high-resolution images from Chandra. In four of these systems, the $15 \AA$ line is suppressed relative to the other Fe XVII lines. For NGC4636 this leads to a turbulent velocity of less than $100 \mathrm{~km} \mathrm{~s}^{-1}$ or less than $5 \%$ for the turbulent to total pressure ratio.

de Plaa et al. (2012) obtained much deeper RGS spectra for two of the ellipticals studied by Werner et al. (2009). Interestingly, in these two systems the amount of turbulence appears 
to be rather high: in NGC5044 turbulent pressure constitutes at least $40 \%$ of the total (with turbulent velocities between $320-720 \mathrm{~km} \mathrm{~s}^{-1}$ ) and in NGC5813 turbulence contributes only $15-45 \%$ to the total pressure, with velocities between $140-540 \mathrm{~km} \mathrm{~s}^{-1}$. However, de Plaa et al. (2012) also point out that the atomic physics of Fe XVII is by no means undisputed, and some of these results depend on the adopted atomic parameters. Fe XVII still remains one of the most difficult and controversial ions, despite its relatively frequent occurrence in $\mathrm{X}$-ray spectra.

The profiles of spectral lines that are affected by resonance scattering will be different from those of non-resonant lines. Because the optical depth in the line core is higher than that in the line wings, the core will be suppressed relative to the wings (e.g. Gilfanov et al. 1987; Shang and Oh 2013). The characteristic depression at the center of the line could therefore be used as a proxy for resonant scattering when observing bright cluster cores.

Apart from the distortions of the line intensity and shape, the resonant scattering causes polarization of the scattered line flux (Sazonov et al. 2002) at the level of 10-15\%. The polarization plane is expected to be perpendicular to the direction towards the cluster center. Gas motions will affect both the degree and the direction of polarization (Zhuravleva et al. 2010). The changes in the polarization signal are in particular sensitive to the gas motions perpendicular to the line of sight. This opens a principal possibility of measuring transverse component of the velocity field, once sensitive X-ray polarimeters with good spectral resolution become available.

\section{Summary and Conclusions}

Between the end of the 90's and the beginning of the 2000's, the X-ray satellites Chandra and XMM-Newton have definitely proven that galaxy clusters are extremely exciting physics laboratory for the characterization of complex plasma processes. In the same years, the development of deep radio observations of clusters have confirmed the presence of a non-thermal intracluster component (cosmic rays and magnetic fields) in the ICM, whose physical properties seem to be strongly connected to the dynamical state and evolutionary history of clusters. As presented in the previous sections, in the next years a new generation of X-ray and radio telescopes will allow us to do a further step forward in the characterization of the MHD physical processes that we expect to be shaping the ICM properties, but for which we are not yet able to make precise measurements.

Great advancements are expected in the direct measurements of turbulence thanks to the increased kinematic resolution of the X-ray satellites ASTRO-H first and Athena then. This will have a relevant impact on both astrophysical and cosmological cluster studies. As described in this paper, turbulent plasma motions are expected to play an important role in the AGN energy dissipation at the center of galaxy clusters and, together with merger induced shocks, in the acceleration of intracluster cosmic rays and in the amplification of magnetic fields. The greatest impact in the characterization of the non-thermal cluster components is expected to come from new and future radio interferometers (SKA in particular and, before, its precursors and pathfinders, such as JVLA, LOFAR, MWA, LWA, ASKAP, MeerKAT, ...; see Norris et al. 2013, and references therein). All together, these instruments will cover a broad spectral range, from a few tens of $\mathrm{MHz}$ to, possibly, $\sim 15 \mathrm{GHz}$. Broadband low-frequency observations at arcsec resolution will be key to systematic searches of steep-spectrum sources, from radio ghosts at the center of clusters, to diffuse radio sources, expected to be hosted in a big fraction of low-mass and minor merging clusters (Cassano et al. 2012). Both total intensity and radio polarization observations from SKA and its 
pathfinders will allow us to access the magnetic field intensity and structure, as well as the acceleration mechanisms responsible for electron acceleration, within the intra- and, possibly, inter-cluster volume up to $z \sim 1$ (e.g. Bonafede et al. 2015; Ferrari et al. 2015; Govoni et al. 2015; Vazza et al. 2015).

Since random turbulent motions are expected to provide a pressure support to the ICM (thus affecting the measure of cluster masses based on the assumption of hydrostatic equilibrium), a precise quantification of the turbulence pressure is not only interesting from the astrophysical point of view, but also crucial for improving the constraints on cosmological parameters through cluster number counts. To this respect, the eROSITA satellite, to be launched in 2016, will survey the sky with unprecedented sensitivity and is expected to detect about $10^{5}$ galaxy clusters down to $5 \times 10^{13} M_{\odot} / h$ and with a median redshift $z \sim 0.35$ (Pillepich et al. 2012). ${ }^{2}$ Later on, the wide field imager onboard of Athena is expected to conduct blind field surveys, allowing to detect and characterize the mass and dynamical state of clusters and groups up to and beyond $z=1$. To be noted that important developments are ongoing also in the (sub-)mm domain, allowing deep improvements in the observations of the Sunyaev-Zel'dovich effect (SZE). ${ }^{3}$

The gamma-ray observations reviewed above have placed stringent limits on the pressure contained in protons of energies above $100 \mathrm{MeV}$ with hard energy spectra. The constraints on the energy density in non-thermal particles with steep enough spectra dominated by particles of energies well below $100 \mathrm{MeV}$ remain to be established with the new low energy gamma-ray experiments which are under development (see e.g. von Ballmoos et al. 2012; Lebrun et al. 2014).

We can conclude that the excellent synergy between future X-ray satellites, together with the huge developments in the new generation of radio and $\mathrm{mm}$ telescopes, will allow us a breakthroughs in our understanding of the evolutionary physics of the intracluster plasma and in the exploitation of galaxy clusters as tools for cosmology.

Acknowledgements We would like to thank the referee for useful comments and the ISSI staff for providing an inspiring atmosphere favorable for intense discussions. We thank Hiroki Akamatsu for providing us with Fig. 21 before publication. W. Forman acknowledges support from NASA contract NASA-03060 that funds the Chandra HRC project, the Chandra archive grant AR1-12007X, and the NASA observing grant GO2-13005X. SRON is financially supported by NWO (the Netherlands Organization for Scientific Research).

\section{References}

M. Ackermann, M. Ajello, A. Albert et al., Search for cosmic-ray-induced gamma-ray emission in galaxy clusters. Astrophys. J. 787, 18 (2014). doi:10.1088/0004-637X/787/1/18

P.A.R. Ade, N. Aghanim, C. Armitage-Caplan, M. Arnaud, M. Ashdown, F. Atrio-Barandela, J. Aumont, H. Aussel, C. Baccigalupi et al., Planck 2013 results. XXIX. The Planck catalogue of SunyaevZeldovich sources. Astron. Astrophys. 571, 29 (2014). doi:10.1051/0004-6361/201321523

H. Akamatsu et al., Astron. Astrophys. (2014, in press)

F. Akimoto, A. Furuzawa, Y. Tawara, K. Yamashita, Iron K-line mapping of clusters of galaxies with the resonance scattering effect. Astron. Nachr. 320, 283 (1999)

\footnotetext{
${ }^{2}$ A flat cosmology with $\Omega_{\Lambda}=1-\Omega_{m}$ and $h=0.701$ is assumed here, see Pillepich et al. (2012).

${ }^{3}$ The SZE is the change in the apparent brightness of the Cosmic Microwave Background radiation towards a cluster of galaxies due to inverse Compton interaction between CMB photons and intracluster electrons., which provide a complementary and powerful tool for detecting clusters and for identifying pressure substructures in their atmospheres (e.g. Korngut et al. 2011; Reichardt et al. 2013; Hasselfield et al. 2013; Ade et al. 2014).
} 
S.W. Allen, R.J.H. Dunn, A.C. Fabian, G.B. Taylor, C.S. Reynolds, The relation between accretion rate and jet power in X-ray luminous elliptical galaxies. Mon. Not. R. Astron. Soc. 372, 21-30 (2006). doi:10.1111/j.1365-2966.2006.10778.x

S.W. Allen, A.E. Evrard, A.B. Mantz, Cosmological parameters from observations of galaxy clusters. Annu. Rev. Astron. Astrophys. 49, 409-470 (2011). doi:10.1146/annurev-astro-081710-102514

E. Amato, P. Blasi, A kinetic approach to cosmic-ray-induced streaming instability at supernova shocks. Mon. Not. R. Astron. Soc. 392, 1591-1600 (2009). doi:10.1111/j.1365-2966.2008.14200.x

N. Asai, N. Fukuda, R. Matsumoto, Three-dimensional MHD simulations of X-ray emitting subcluster plasmas in cluster of galaxies. Adv. Space Res. 36, 636-642 (2005). doi:10.1016/j.asr.2005.04.041

Y. Ascasibar, M. Markevitch, The origin of cold fronts in the cores of relaxed galaxy clusters. Astrophys. J. 650, 102-127 (2006). doi:10.1086/506508

A. Baldi, S. Ettori, S. Molendi, I. Balestra, F. Gastaldello, P. Tozzi, An XMM-Newton spatially-resolved study of metal abundance evolution in distant galaxy clusters. Astron. Astrophys. 537, 142 (2012). doi:10.1051/0004-6361/201117836

A.M. Beck, K. Dolag, H. Lesch, P.P. Kronberg, Strong magnetic fields and large rotation measures in protogalaxies from supernova seeding. Mon. Not. R. Astron. Soc. 435, 3575-3586 (2013). doi:10.1093/ mnras/stt1549

A.R. Bell, Turbulent amplification of magnetic field and diffusive shock acceleration of cosmic rays. Mon. Not. R. Astron. Soc. 353, 550-558 (2004). doi:10.1111/j.1365-2966.2004.08097.x

V.S. Berezinsky, P. Blasi, V.S. Ptuskin, Clusters of galaxies as storage room for cosmic rays. Astrophys. J. 487, 529-535 (1997)

S. Bertone, C. Vogt, T. Enßlin, Magnetic field seeding by galactic winds. Mon. Not. R. Astron. Soc. 370, 319-330 (2006). doi:10.1111/j.1365-2966.2006.10474.x

J. Binney, G. Tabor, Evolving cooling flows. Mon. Not. R. Astron. Soc. 276, 663 (1995)

L. Bîrzan, D.A. Rafferty, P.E.J. Nulsen, B.R. McNamara, H.J.A. Röttgering, M.W. Wise, R. Mittal, The duty cycle of radio-mode feedback in complete samples of clusters. Mon. Not. R. Astron. Soc. 427, 34683488 (2012). doi:10.1111/j.1365-2966.2012.22083.x

P. Blasi, S. Colafrancesco, Cosmic rays, radio halos and nonthermal X-ray emission in clusters of galaxies. Astropart. Phys. 12, 169-183 (1999). doi:10.1016/S0927-6505(99)00079-1

H. Boehringer, W. Voges, A.C. Fabian, A.C. Edge, D.M. Neumann, A ROSAT HRI study of the interaction of the X-ray-emitting gas and radio lobes of NGC 1275. Mon. Not. R. Astron. Soc. 264, 25-28 (1993)

H. Böhringer, N. Werner, X-ray spectroscopy of galaxy clusters: Studying astrophysical processes in the largest celestial laboratories. Astron. Astrophys. Rev. 18, 127-196 (2010). doi:10.1007/s00159-0090023-3

H. Bohringer, P.E.J. Nulsen, R. Braun, A.C. Fabian, The interaction of the radio halo of M87 with the cooling intracluster medium of the Virgo cluster. Mon. Not. R. Astron. Soc. 274, 67-71 (1995)

H. Böhringer, K. Matsushita, E. Churazov, Y. Ikebe, Y. Chen, The new emerging model for the structure of cooling cores in clusters of galaxies. Astron. Astrophys. 382, 804-820 (2002). doi:10.1051/00046361:20011708

A. Bonafede, F. Govoni, L. Feretti, M. Murgia, G. Giovannini, M. Brüggen, Fractional polarization as a probe of magnetic fields in the intra-cluster medium. Astron. Astrophys. 530, 24 (2011). doi:10.1051/0004$6361 / 201016298$

A. Bonafede, F. Vazza, M. Brüggen, T. Akahori, E. Carretti, S. Colafrancesco, L. Feretti, C. Ferrari, G. Giovannini, F. Govoni, M. Johnston-Hollitt, M. Murgia, L. Rudnick, A. Scaife, V. Vacca, Unravelling the origin of large-scale magnetic fields in galaxy clusters and beyond, in Proceedings of Advancing Astrophysics with the Square Kilometre Array, PoS(AASKA14)105 (2015)

H. Bondi, On spherically symmetrical accretion. Mon. Not. R. Astron. Soc. 112, 195 (1952)

R.G. Bower, A.J. Benson, R. Malbon, J.C. Helly, C.S. Frenk, C.M. Baugh, S. Cole, C.G. Lacey, Breaking the hierarchy of galaxy formation. Mon. Not. R. Astron. Soc. 370, 645-655 (2006). doi:10.1111/j.13652966.2006.10519.x

M. Brüggen, A. Bykov, D. Ryu, H. Röttgering, Magnetic fields, relativistic particles, and shock waves in cluster outskirts. Space Sci. Rev. 166, 187-213 (2012). doi:10.1007/s11214-011-9785-9

G. Brunetti, T.W. Jones, Cosmic rays in galaxy clusters and their nonthermal emission. Int. J. Mod. Phys. D 23, 30007 (2014). doi:10.1142/S0218271814300079

G. Brunetti, A. Lazarian, Acceleration of primary and secondary particles in galaxy clusters by compressible MHD turbulence: From radio haloes to gamma-rays. Mon. Not. R. Astron. Soc. 410, 127-142 (2011). doi:10.1111/j.1365-2966.2010.17457.x

G. Brunetti, G. Setti, L. Feretti, G. Giovannini, Particle reacceleration in the Coma cluster: Radio properties and hard X-ray emission. Mon. Not. R. Astron. Soc. 320, 365 (2001). doi:10.1046/j.1365-8711.2001. 03978.x 
E. Bulbul, R.K. Smith, M. Loewenstein, A new method to constrain supernova fractions using X-ray observations of clusters of galaxies. Astrophys. J. 753, 54 (2012). doi:10.1088/0004-637X/753/1/54

A.M. Bykov, Particle acceleration and nonthermal phenomena in superbubbles. Space Sci. Rev. 99, 317-326 (2001)

A.M. Bykov, Nonthermal particles and photons in starburst regions and superbubbles. Astron. Astrophys. Rev. 22, 22-77 (2014). doi:10.1007/s00159-014-0077-8

A.M. Bykov, I. Toptygin, Particle kinetics in highly turbulent plasmas (renormalization and self-consistent field methods). Phys. Usp. 36, 1020-1052 (1993). doi:10.1070/PU1993v036n11ABEH002179

A.M. Bykov, Y.A. Uvarov, Electron kinetics in collisionless shock waves. Sov. Phys. JETP 88, 465-475 (1999). doi:10.1134/1.558817

A.M. Bykov, K. Dolag, F. Durret, Cosmological shock waves. Space Sci. Rev. 134, 119-140 (2008). doi:10. 1007/s11214-008-9312-9

A.M. Bykov, D.C. Ellison, M. Renaud, Magnetic fields in cosmic particle acceleration sources. Space Sci. Rev. 166, 71-95 (2012). doi:10.1007/s11214-011-9761-4

A.M. Bykov, D.C. Ellison, S.M. Osipov, A.E. Vladimirov, Magnetic field amplification in nonlinear diffusive shock acceleration including resonant and non-resonant cosmic-ray driven instabilities. Astrophys. J. 789, 137 (2014). doi:10.1088/0004-637X/789/2/137

R. Cassano, G. Brunetti, R.P. Norris, H.J.A. Röttgering, M. Johnston-Hollitt, M. Trasatti, Radio halos in future surveys in the radio continuum. Astron. Astrophys. 548, 100 (2012). doi:10.1051/0004-6361/ 201220018

S.K. Chakrabarti, R. Rosner, S.I. Vainshtein, Possible role of massive black holes in the generation of galactic magnetic fields. Nature 368, 434-436 (1994). doi:10.1038/368434a0

E. Churazov, W. Forman, C. Jones, H. Böhringer, Asymmetric, arc minute scale structures around NGC 1275. Astron. Astrophys. 356, 788-794 (2000)

E. Churazov, M. Brüggen, C.R. Kaiser, H. Böhringer, W. Forman, Evolution of buoyant bubbles in M87. Astrophys. J. 554, 261-273 (2001). doi:10.1086/321357

E. Churazov, R. Sunyaev, W. Forman, H. Böhringer, Cooling flows as a calorimeter of active galactic nucleus mechanical power. Mon. Not. R. Astron. Soc. 332, 729-734 (2002). doi:10.1046/j.1365-8711.2002. 05332.x

E. Churazov, W. Forman, C. Jones, H. Böhringer, XMM-Newton observations of the Perseus cluster. I. The temperature and surface brightness structure. Astrophys. J. 590, 225-237 (2003). doi:10.1086/374923

E. Churazov, W. Forman, C. Jones, R. Sunyaev, H. Böhringer, XMM-Newton observations of the Perseus cluster-II. Evidence for gas motions in the core. Mon. Not. R. Astron. Soc. 347, 29-35 (2004). doi:10. 1111/j.1365-2966.2004.07201.x

E. Churazov, S. Sazonov, R. Sunyaev, W. Forman, C. Jones, H. Böhringer, Supermassive black holes in elliptical galaxies: Switching from very bright to very dim. Mon. Not. R. Astron. Soc. 363, 91-95 (2005). doi:10.1111/j.1745-3933.2005.00093.x

E. Churazov, I. Zhuravleva, S. Sazonov, R. Sunyaev, Resonant scattering of X-ray emission lines in the hot intergalactic medium. Space Sci. Rev. 157, 193-209 (2010). doi:10.1007/s11214-010-9685-4

E. Churazov, A. Vikhlinin, I. Zhuravleva, A. Schekochihin, I. Parrish, R. Sunyaev, W. Forman, H. Böhringer, S. Randall, X-ray surface brightness and gas density fluctuations in the Coma cluster. Mon. Not. R. Astron. Soc. 421, 1123-1135 (2012). doi:10.1111/j.1365-2966.2011.20372.x

L.L. Cowie, J. Binney, Radiative regulation of gas flow within clusters of galaxies-A model for cluster X-ray sources. Astrophys. J. 215, 723-732 (1977). doi:10.1086/155406

D.J. Croton, V. Springel, S.D.M. White, G. De Lucia, C.S. Frenk, L. Gao, A. Jenkins, G. Kauffmann, J.F. Navarro, N. Yoshida, The many lives of active galactic nuclei: Cooling flows, black holes and the luminosities and colours of galaxies. Mon. Not. R. Astron. Soc. 365, 11-28 (2006). doi:10.1111/j.13652966.2005.09675.x

L.P. David, P.E.J. Nulsen, B.R. McNamara, W. Forman, C. Jones, T. Ponman, B. Robertson, M. Wise, A highresolution study of the hydra a cluster with Chandra: Comparison of the core mass distribution with theoretical predictions and evidence for feedback in the cooling flow. Astrophys. J. 557, 546-559 (2001). doi: $10.1086 / 322250$

F. de Gasperin, E. Orrú, M. Murgia et al., M 87 at metre wavelengths: The LOFAR picture. Astron. Astrophys. 547, 56 (2012). doi:10.1051/0004-6361/201220209

S. de Grandi, S. Molendi, Metal abundances in the cool cores of galaxy clusters. Astron. Astrophys. 508, 565 (2009). doi:10.1051/0004-6361/200912745

J. de Plaa, The origin of the chemical elements in cluster cores. Astron. Nachr. 334, 416 (2013). doi:10.1002/ asna.201211870

J. de Plaa, N. Werner, A.M. Bykov, J.S. Kaastra, M. Méndez, J. Vink, J.A.M. Bleeker, M. Bonamente, J.R. Peterson, Chemical evolution in Sérsic 159-03 observed with XMM-Newton. Astron. Astrophys. 452, 397 (2006). doi:10.1051/0004-6361:20053864 
J. de Plaa, N. Werner, J.A.M. Bleeker, J. Vink, J.S. Kaastra, M. Méndez, Constraining supernova models using the hot gas in clusters of galaxies. Astron. Astrophys. 465, 345 (2007). doi:10.1051/0004-6361: 20066382

J. de Plaa, I. Zhuravleva, N. Werner, J.S. Kaastra, E. Churazov, R.K. Smith, A.J.J. Raassen, Y.G. Grange, Estimating turbulent velocities in the elliptical galaxies NGC 5044 and NGC 5813. Astron. Astrophys. 539, 34 (2012). doi:10.1051/0004-6361/201118404

R. della Ceca, R.E. Griffiths, T.M. Heckman, J.W. MacKenty, ASCA observations of starbursting dwarf galaxies: The case of NGC 1569. Astrophys. J. 469, 662 (1996). doi:10.1086/177813

B. Dennison, Formation of radio halos in clusters of galaxies from cosmic-ray protons. Astrophys. J. Lett. 239, 93-96 (1980). doi:10.1086/183300

T. Di Matteo, S.W. Allen, A.C. Fabian, A.S. Wilson, A.J. Young, Accretion onto the supermassive black hole in M87. Astrophys. J. 582, 133-140 (2003). doi:10.1086/344504

K. Dolag, A.M. Bykov, A. Diaferio, Non-thermal processes in cosmological simulations. Space Sci. Rev. 134, 311-335 (2008). doi:10.1007/s11214-008-9319-2

J. Donnert, K. Dolag, H. Lesch, E. Müller, Cluster magnetic fields from galactic outflows. Mon. Not. R. Astron. Soc. 392, 1008-1021 (2009). doi:10.1111/j.1365-2966.2008.14132.x

J. Donnert, K. Dolag, G. Brunetti, R. Cassano, A. Bonafede, Radio haloes from simulations and hadronic models-I. The Coma cluster. Mon. Not. R. Astron. Soc. 401, 47-54 (2010). doi:10.1111/j.1365-2966. 2009.15655.x

Y. Dubois, R. Teyssier, Magnetised winds in dwarf galaxies. Astron. Astrophys. 523, 72 (2010). doi:10. 1051/0004-6361/200913014

R.J.H. Dunn, A.C. Fabian, Investigating AGN heating in a sample of nearby clusters. Mon. Not. R. Astron. Soc. 373, 959-971 (2006). doi:10.1111/j.1365-2966.2006.11080.x

D. Eckert, S. Molendi, F. Vazza, S. Ettori, S. Paltani, The X-ray/SZ view of the virial region. I. Thermodynamic properties. Astron. Astrophys. 551, 22 (2013). doi:10.1051/0004-6361/201220402

D. Eckert, S. Molendi, M. Owers, M. Gaspari, T. Venturi, L. Rudnick, S. Ettori, S. Paltani, F. Gastaldello, M. Rossetti, The stripping of a galaxy group diving into the massive cluster A2142. Astron. Astrophys. 570, 119 (2014). doi:10.1051/0004-6361/201424259

T.A. Enßlin, S. Heinz, Radio and X-ray detectability of buoyant radio plasma bubbles in clusters of galaxies. Astron. Astrophys. 384, 27-30 (2002). doi:10.1051/0004-6361:20020207

T. Enßlin, C. Pfrommer, F. Miniati, K. Subramanian, Cosmic ray transport in galaxy clusters: Implications for radio halos, gamma-ray signatures, and cool core heating. Astron. Astrophys. 527, 99 (2011). doi:10.1051/0004-6361/201015652

S. Ettori, A.C. Fabian, Chandra constraints on the thermal conduction in the intracluster plasma of A2142. Mon. Not. R. Astron. Soc. 317, 57-59 (2000). doi:10.1046/j.1365-8711.2000.03899.x

A.C. Fabian, Cooling flows in clusters of galaxies. Annu. Rev. Astron. Astrophys. 32, 277-318 (1994). doi:10.1146/annurev.aa.32.090194.001425

A.C. Fabian, Observational evidence of active galactic nuclei feedback. Annu. Rev. Astron. Astrophys. 50, 455-489 (2012). doi:10.1146/annurev-astro-081811-125521

A.C. Fabian, P.E.J. Nulsen, Subsonic accretion of cooling gas in clusters of galaxies. Mon. Not. R. Astron. Soc. 180, 479-484 (1977)

A.C. Fabian, J.S. Sanders, S. Ettori, G.B. Taylor, S.W. Allen, C.S. Crawford, K. Iwasawa, R.M. Johnstone, P.M. Ogle, Chandra imaging of the complex X-ray core of the Perseus cluster. Mon. Not. R. Astron. Soc. 318, 65-68 (2000). doi:10.1046/j.1365-8711.2000.03904.x

A.C. Fabian, J.S. Sanders, C.S. Crawford, C.J. Conselice, J.S. Gallagher, R.F.G. Wyse, The relationship between the optical $\mathrm{H} \alpha$ filaments and the X-ray emission in the core of the Perseus cluster. Mon. Not. R. Astron. Soc. 344, 48-52 (2003). doi:10.1046/j.1365-8711.2003.06856.x

A.C. Fabian, J.S. Sanders, G.B. Taylor, S.W. Allen, C.S. Crawford, R.M. Johnstone, K. Iwasawa, A very deep Chandra observation of the Perseus cluster: Shocks, ripples and conduction. Mon. Not. R. Astron. Soc. 366, 417-428 (2006). doi:10.1111/j.1365-2966.2005.09896.x

L. Feretti, G. Giovannini, F. Govoni, M. Murgia, Clusters of galaxies: Observational properties of the diffuse radio emission. Astron. Astrophys. Rev. 20, 54 (2012). doi:10.1007/s00159-012-0054-Z

L. Ferrarese, D. Merritt, A fundamental relation between supermassive black holes and their host galaxies. Astrophys. J. Lett. 539, 9-12 (2000). doi:10.1086/312838

C. Ferrari, F. Govoni, S. Schindler, A.M. Bykov, Y. Rephaeli, Observations of extended radio emission in clusters. Space Sci. Rev. 134, 93-118 (2008). doi:10.1007/s11214-008-9311-x

C. Ferrari, A. Dabbech, O. Smirnov et al., Non-thermal emission from galaxy clusters: Feasibility study with SKA1, in Proceedings of Advancing Astrophysics with the Square Kilometre Array, PoS(AASKA14)105 (2015) 
W. Forman, P. Nulsen, S. Heinz, F. Owen, J. Eilek, A. Vikhlinin, M. Markevitch, R. Kraft, E. Churazov, C. Jones, Reflections of active galactic nucleus outbursts in the gaseous atmosphere of M87. Astrophys. J. 635, 894-906 (2005). doi:10.1086/429746

W. Forman, C. Jones, E. Churazov, M. Markevitch, P. Nulsen, A. Vikhlinin, M. Begelman, H. Böhringer, J. Eilek, S. Heinz, R. Kraft, F. Owen, M. Pahre, Filaments, bubbles, and weak shocks in the gaseous atmosphere of M87. Astrophys. J. 665, 1057-1066 (2007). doi:10.1086/519480

Y. Fujita, T. Matsumoto, K. Wada, Strong turbulence in the cool cores of galaxy clusters: Can tsunamis solve the cooling flow problem? Astrophys. J. Lett. 612, 9-12 (2004). doi:10.1086/424483

J.M. Gabor, R. Davé, K. Finlator, B.D. Oppenheimer, How is star formation quenched in massive galaxies? Mon. Not. R. Astron. Soc. 407, 749 (2010). doi:10.1111/j.1365-2966.2010.16961.x

E. Gallo, R.P. Fender, G.G. Pooley, A universal radio-X-ray correlation in low/hard state black hole binaries. Mon. Not. R. Astron. Soc. 344, 60-72 (2003). doi:10.1046/j.1365-8711.2003.06791.x

M. Gaspari, M. Ruszkowski, P. Sharma, Cause and effect of feedback: Multiphase gas in cluster cores heated by AGN jets. Astrophys. J. 746, 94 (2012). doi:10.1088/0004-637X/746/1/94

F. Gastaldello, S. Molendi, Ni abundance in the core of the Perseus cluster: An answer to the significance of resonant scattering. Astrophys. J. 600, 670-680 (2004). doi:10.1086/379970

K. Gebhardt, R. Bender, G. Bower, A. Dressler, S.M. Faber, A.V. Filippenko, R. Green, C. Grillmair, L.C. Ho, J. Kormendy, T.R. Lauer, J. Magorrian, J. Pinkney, D. Richstone, S. Tremaine, A relationship between nuclear black hole mass and galaxy velocity dispersion. Astrophys. J. Lett. 539, 13-16 (2000). doi:10. $1086 / 312840$

S. Ghizzardi, M. Rossetti, S. Molendi, Cold fronts in galaxy clusters. Astron. Astrophys. 516, 32 (2010). doi:10.1051/0004-6361/200912496

S. Giacintucci, M. Markevitch, G. Brunetti, J.A. ZuHone, T. Venturi, P. Mazzotta, H. Bourdin, Mapping the particle acceleration in the cool core of the galaxy cluster RX J1720.1+2638. Astrophys. J. 795, 73 (2014). doi:10.1088/0004-637X/795/1/73

M.R. Gilfanov, R.A. Syunyaev, E.M. Churazov, Radial brightness profiles of resonance X-ray lines in galaxy clusters. Sov. Astron. Lett. 13, 3 (1987)

F. Govoni, M. Murgia, H. Xu, H. Li, M.L. Norman, L. Feretti, G. Giovannini, V. Vacca, Polarization of cluster radio halos with upcoming radio interferometers. Astron. Astrophys. 554, 102 (2013). doi:10.1051/ 0004-6361/201321403

F. Govoni, M. Murgia, H. Xu et al., Cluster magnetic fields through the study of polarized radio halos, in Proceedings of Advancing Astrophysics with the Square Kilometre Array, PoS(AASKA14)105 (2015)

Y.G. Grange, J. de Plaa, J.S. Kaastra, N. Werner, F. Verbunt, F. Paerels, C.P. de Vries, The metal contents of two groups of galaxies. Astron. Astrophys. 531, 15 (2011). doi:10.1051/0004-6361/201016187

S.F. Gull, K.J.E. Northover, Bubble model of extragalactic radio sources. Nature 244, 80-83 (1973). doi:10. 1038/244080a0

X. Guo, L. Sironi, R. Narayan, Non-thermal electron acceleration in low mach number collisionless shocks. I. Particle energy spectra and acceleration mechanism. Astrophys. J. 794, 153 (2014a). doi:10.1088/ 0004-637X/794/2/153

X. Guo, L. Sironi, R. Narayan, Non-thermal electron acceleration in low mach number collisionless shocks. II. Firehose-mediated Fermi acceleration and its dependence on pre-shock conditions. Astrophys. J. 797, 47 (2014b). doi:10.1088/0004-637X/797/1/47

M. Hasselfield, M. Hilton, T.A. Marriage et al., The Atacama cosmology telescope: Sunyaev-Zel'dovich selected galaxy clusters at $148 \mathrm{GHz}$ from three seasons of data. J. Cosmol. Astropart. Phys. 7, 8 (2013). doi:10.1088/1475-7516/2013/07/008

D.C. Hines, J.A. Eilek, F.N. Owen, Filaments in the radio lobes of M87. Astrophys. J. 347, 713-726 (1989). doi:10.1086/168163

J. Hlavacek-Larrondo, A.C. Fabian, A.C. Edge, H. Ebeling, J.S. Sanders, M.T. Hogan, G.B. Taylor, Extreme AGN feedback in the MAssive cluster survey: A detailed study of X-ray cavities at $z>0.3$. Mon. Not. R. Astron. Soc. 421, 1360-1384 (2012). doi:10.1111/j.1365-2966.2011.20405.x

D.A. Howell, Type Ia supernovae as stellar endpoints and cosmological tools. Nat. Commun. 2, 350 (2011). doi: $10.1038 /$ ncomms 1344

S. Ichimaru, Bimodal behavior of accretion disks - Theory and application to Cygnus X-1 transitions. Astrophys. J. 214, 840-855 (1977). doi:10.1086/155314

N.A. Inogamov, R.A. Sunyaev, Turbulence in clusters of galaxies and X-ray line profiles. Astron. Lett. 29, 791-824 (2003). doi:10.1134/1.1631412

K. Iwamoto, F. Brachwitz, K. Nomoto, N. Kishimoto, H. Umeda, W.R. Hix, F.K. Thielemann, Nucleosynthesis in Chandrasekhar mass models for type IA supernovae and constraints on progenitor systems and burning- front propagation. Astrophys. J. Suppl. Ser. 125, 439 (1999). doi:10.1086/313278

F.C. Jones, J.R. Jokipii, M.G. Baring, Charged-particle motion in electromagnetic fields having at least one ignorable spatial coordinate. Astrophys. J. 509, 238-243 (1998). doi:10.1086/306480 
J.S. Kaastra, A.M. Bykov, N. Werner, Non-Maxwellian electron distributions in clusters of galaxies. Astron. Astrophys. 503, 373-378 (2009). doi:10.1051/0004-6361/200912492

A.I. Karakas, Updated stellar yields from asymptotic giant branch models. Mon. Not. R. Astron. Soc. 403, 1413 (2010). doi:10.1111/j.1365-2966.2009.16198.x

C.F. Kennel, J.P. Edmiston, T. Hada, A quarter century of collisionless shock research, in Washington DC American Geophysical Union Geophysical Monograph Series, vol. 34 (1985), pp. 1-36

A.A. Kepley, S. Mühle, J. Everett, E.G. Zweibel, E.M. Wilcots, U. Klein, The role of the magnetic field in the interstellar medium of the post-starburst dwarf irregular galaxy NGC 1569. Astrophys. J. 712, 536-557 (2010). doi:10.1088/0004-637X/712/1/536

U. Keshet, M. Markevitch, Y. Birnboim, A. Loeb, Dynamics and magnetization in galaxy cluster cores traced by X-ray cold fronts. Astrophys. J. Lett. 719, 74-78 (2010). doi:10.1088/2041-8205/719/1/L74

U. Klein, E. Hummel, D.J. Bomans, U. Hopp, The synchrotron halo and magnetic field of NGC 4449. Astron. Astrophys. 313, 396-404 (1996)

P.M. Korngut, S.R. Dicker, E.D. Reese, B.S. Mason, M.J. Devlin, T. Mroczkowski, C.L. Sarazin, M. Sun, J. Sievers, MUSTANG high angular resolution Sunyaev-Zel'dovich effect imaging of substructure in four galaxy clusters. Astrophys. J. 734, 10 (2011). doi:10.1088/0004-637X/734/1/10

A.V. Kravtsov, S. Borgani, Formation of galaxy clusters. Annu. Rev. Astron. Astrophys. 50, 353-409 (2012). doi:10.1146/annurev-astro-081811-125502

P.P. Kronberg, H. Lesch, U. Hopp, Magnetization of the intergalactic medium by primeval galaxies. Astrophys. J. 511, 56-64 (1999). doi:10.1086/306662

S.M. Lea, The dynamics of the intergalactic medium in the vicinity of clusters of galaxies. Astrophys. J. 203, 569-580 (1976). doi:10.1086/154113

F. Lebrun, R. Terrier, P. Laurent et al., The gamma cube: A novel concept of gamma-ray telescope, in Society of Photo-Optical Instrumentation Engineers (SPIE) Conference Series, vol. 9144 (2014). doi:10.1117/ 12.2055951

M. Lyutikov, Magnetic draping of merging cores and radio bubbles in clusters of galaxies. Mon. Not. R. Astron. Soc. 373, 73-78 (2006). doi:10.1111/j.1365-2966.2006.10835.x

M. Machacek, A. Dosaj, W. Forman, C. Jones, M. Markevitch, A. Vikhlinin, A. Warmflash, R. Kraft, Infall of the elliptical galaxy NGC 1404 into the fornax cluster. Astrophys. J. 621, 663-672 (2005). doi:10. $1086 / 427548$

M. Markevitch, Chandra observation of the most interesting cluster in the universe, in The X-Ray Universe 2005, ed. by A. Wilson. ESA Special Publication, vol. 604 (2006), p. 723

M. Markevitch, A. Vikhlinin, Shocks and cold fronts in galaxy clusters. Phys. Rep. 443, 1-53 (2007). doi:10.1016/j.physrep.2007.01.001

M. Markevitch, T.J. Ponman, P.E.J. Nulsen, M.W. Bautz, D.J. Burke, L.P. David, D. Davis, R.H. Donnelly, W.R. Forman, C. Jones, J. Kaastra, E. Kellogg, D.W. Kim, J. Kolodziejczak, P. Mazzotta, A. Pagliaro, S. Patel, L. Van Speybroeck, A. Vikhlinin, J. Vrtilek, M. Wise, P. Zhao Chandra, Observation of Abell 2142: Survival of dense subcluster cores in a merger. Astrophys. J. 541, 542-549 (2000). doi:10.1086/ 309470

M. Markevitch, A. Vikhlinin, P. Mazzotta, Nonhydrostatic gas in the core of the relaxed galaxy cluster A1795. Astrophys. J. Lett. 562, 153-156 (2001). doi:10.1086/337973

M. Markevitch, A. Vikhlinin, W.R. Forman, A. High, Resolution picture of the intracluster gas, in Matter and Energy in Clusters of Galaxies, ed. by S. Bowyer, C.Y. Hwang. Astronomical Society of the Pacific Conference Series, vol. 301 (2003), p. 37

C.L. Martin, The impact of star formation on the interstellar medium in dwarf galaxies. II. The formation of galactic winds. Astrophys. J. 506, 222-252 (1998). doi:10.1086/306219

F. Matteucci, F. Calura, Early chemical enrichment of the universe and the role of very massive population III stars. Mon. Not. R. Astron. Soc. 360, 447 (2005). doi:10.1111/j.1365-2966.2005.08908.x

P. Mazzotta, M. Markevitch, A. Vikhlinin, W.R. Forman, L.P. David, L. van Speybroeck Chandra, Observation of RX J1720.1+2638: A nearly relaxed cluster with a fast-moving core? Astrophys. J. 555, 205-214 (2001). doi:10.1086/321484

M. McDonald, M. Bayliss, B.A. Benson et al., A massive, cooling-flow-induced starburst in the core of a luminous cluster of galaxies. Nature 488, 349-352 (2012). doi:10.1038/nature11379

M. McDonald, B.A. Benson, A. Vikhlinin et al., The growth of cool cores and evolution of cooling properties in a sample of 83 galaxy clusters at $0.3<z<1.2$ selected from the SPT-SZ survey. Astrophys. J. 774, 23 (2013). doi:10.1088/0004-637X/774/1/23

B.R. McNamara, P.E.J. Nulsen, Heating hot atmospheres with active galactic nuclei. Annu. Rev. Astron. Astrophys. 45, 117-175 (2007). doi:10.1146/annurev.astro.45.051806.110625

B.R. McNamara, M. Wise, P.E.J. Nulsen, L.P. David, C.L. Sarazin, M. Bautz, M. Markevitch, A. Vikhlinin, W.R. Forman, C. Jones, D.E. Harris, Chandra X-ray observations of the hydra a cluster: An interaction 
between the radio source and the X-ray-emitting gas. Astrophys. J. Lett. 534, 135-138 (2000). doi:10. 1086/312662

B.R. McNamara, P.E.J. Nulsen, M.W. Wise, D.A. Rafferty, C. Carilli, C.L. Sarazin, E.L. Blanton, The heating of gas in a galaxy cluster by X-ray cavities and large-scale shock fronts. Nature 433, 45-47 (2005). doi:10.1038/nature03202

G. Miley, The structure of extended extragalactic radio sources. Annu. Rev. Astron. Astrophys. 18, 165-218 (1980). doi:10.1146/annurev.aa.18.090180.001121

E.T. Million, N. Werner, A. Simionescu, S.W. Allen, P.E.J. Nulsen, A.C. Fabian, H. Böhringer, J.S. Sanders, Feedback under the microscope-I. Thermodynamic structure and AGN-driven shocks in M87. Mon. Not. R. Astron. Soc. 407, 2046-2062 (2010). doi:10.1111/j.1365-2966.2010.17220.x

S. Molendi, G. Matt, L.A. Antonelli, F. Fiore, R. Fusco-Femiano, J. Kaastra, C. Maccarone, C. Perola, How abundant is iron in the core of the Perseus cluster? Astrophys. J. 499, 608-613 (1998)

R. Mushotzky, M. Loewenstein, K.A. Arnaud, T. Tamura, Y. Fukazawa, K. Matsushita, K. Kikuchi, I. Hatsukade, Measurement of the elemental abundances in four rich clusters of galaxies. I. Observations. Astrophys. J. 466, 686 (1996). doi:10.1086/177541

R. Narayan, I. Yi, Advection-dominated accretion: A self-similar solution. Astrophys. J. Lett. 428, 13-16 (1994). doi:10.1086/187381

R.P. Norris, J. Afonso, D. Bacon et al., Radio continuum surveys with square kilometre array pathfinders. Publ. Astron. Soc. Aust. 30, 20 (2013). doi:10.1017/pas.2012.020

P. Nulsen, E. Roediger, Sloshing cold fronts \& cluster G-modes, in SnowCLUSTER 2013, Physics of Galaxy Clusters (2013), p. 68

G.A. Ogrean, M. Brüggen, R.J. van Weeren, A. Burgmeier, A. Simionescu, No shock across part of a radio relic in the merging galaxy cluster ZwCl 2341.1+0000? Mon. Not. R. Astron. Soc. 443, 2463-2474 (2014). doi:10.1093/mnras/stu1299

H. Omma, J. Binney, G. Bryan, A. Slyz, Heating cooling flows with jets. Mon. Not. R. Astron. Soc. 348, 1105-1119 (2004). doi:10.1111/j.1365-2966.2004.07382.x

F.N. Owen, J.A. Eilek, N.E. Kassim, M87 at 90 centimeters: A different picture. Astrophys. J. 543, 611-619 (2000). doi:10.1086/317151

A. Pedlar, H.S. Ghataure, R.D. Davies, B.A. Harrison, R. Perley, P.C. Crane, S.W. Unger, The radio structure of NGC1275. Mon. Not. R. Astron. Soc. 246, 477 (1990)

C.B. Peres, A.C. Fabian, A.C. Edge, S.W. Allen, R.M. Johnstone, D.A. White, A ROSAT study of the cores of clusters of galaxies-I. Cooling flows in an X-ray flux-limited sample. Mon. Not. R. Astron. Soc. 298, 416-432 (1998). doi:10.1046/j.1365-8711.1998.01624.x

J.R. Peterson, A.C. Fabian, X-ray spectroscopy of cooling clusters. Phys. Rep. 427, 1-39 (2006). doi:10. 1016/j.physrep.2005.12.007

V. Petrosian, A. Bykov, Y. Rephaeli, Nonthermal radiation mechanisms. Space Sci. Rev. 134, 191-206 (2008). doi:10.1007/s11214-008-9327-2

A. Pillepich, C. Porciani, T.H. Reiprich, The X-ray cluster survey with eRosita: Forecasts for cosmology, cluster physics and primordial non-Gaussianity. Mon. Not. R. Astron. Soc. 422, 44-69 (2012). doi:10. 1111/j.1365-2966.2012.20443.x

F. Pizzolato, N. Soker, On the nature of feedback heating in cooling flow clusters. Astrophys. J. 632, 821-830 (2005). doi:10.1086/444344

S. Planelles, D.R.G. Schleicher, A.M. Bykov, Large-scale structure formation: From the first non-linear objects to massive galaxy clusters. Space Sci. Rev. (2014). doi:10.1007/s11214-014-0045-7

D.A. Prokhorov, E.M. Churazov, Counting gamma rays in the directions of galaxy clusters. Astron. Astrophys. 567, 93 (2014). doi:10.1051/0004-6361/201322454

D.A. Rafferty, B.R. McNamara, P.E.J. Nulsen, M.W. Wise, The feedback-regulated growth of black holes and bulges through gas accretion and starbursts in cluster central dominant galaxies. Astrophys. J. 652, 216-231 (2006). doi:10.1086/507672

S.W. Randall, W.R. Forman, S. Giacintucci, P.E.J. Nulsen, M. Sun, C. Jones, E. Churazov, L.P. David, R. Kraft, M. Donahue, E.L. Blanton, A. Simionescu, N. Werner, Shocks and cavities from multiple outbursts in the galaxy group NGC 5813: A window to active galactic nucleus feedback. Astrophys. J. 726, 86 (2011). doi:10.1088/0004-637X/726/2/86

M.J. Rees, The origin and cosmogonic implications of seed magnetic fields. Q. J. R. Astron. Soc. 28, $197-206$ (1987)

M.J. Rees, M.C. Begelman, R.D. Blandford, E.S. Phinney, Ion-supported tori and the origin of radio jets. Nature 295, 17-21 (1982). doi:10.1038/295017a0

C.L. Reichardt, B. Stalder, L.E. Bleem et al., Galaxy clusters discovered via the Sunyaev-Zel'dovich effect in the first 720 square degrees of the South pole telescope survey. Astrophys. J. 763, 127 (2013). doi:10. 1088/0004-637X/763/2/127 
T.H. Reiprich, K. Basu, S. Ettori, H. Israel, L. Lovisari, S. Molendi, E. Pointecouteau, M. Roncarelli, Outskirts of galaxy clusters. Space Sci. Rev. 177, 195-245 (2013). doi:10.1007/s11214-013-9983-8

E. Roediger, R.P. Kraft, W.R. Forman, P.E.J. Nulsen, E. Churazov, Kelvin-Helmholtz instabilities at the sloshing cold fronts in the Virgo cluster as a measure for the effective intracluster medium viscosity. Astrophys. J. 764, 60 (2013). doi:10.1088/0004-637X/764/1/60

D. Romano, A.I. Karakas, M. Tosi, F. Matteucci, Quantifying the uncertainties of chemical evolution studies. II. Stellar yields. Astron. Astrophys. 522, 32 (2010). doi:10.1051/0004-6361/201014483

M. Rossetti, D. Eckert, S. De Grandi, F. Gastaldello, S. Ghizzardi, E. Roediger, S. Molendi, Abell 2142 at large scales: An extreme case for sloshing? Astron. Astrophys. 556, 44 (2013). doi:10.1051/0004-6361/ 201321319

D. Ryu, H. Kang, E. Hallman, T.W. Jones, Cosmological shock waves and their role in the large-scale structure of the universe. Astrophys. J. 593, 599-610 (2003). doi:10.1086/376723

J.S. Sanders, A.C. Fabian, R.K. Smith, Constraints on turbulent velocity broadening for a sample of clusters, groups and elliptical galaxies using XMM-Newton. Mon. Not. R. Astron. Soc. 410, 1797-1812 (2011). doi:10.1111/j.1365-2966.2010.17561.x

K. Sato, K. Tokoi, K. Matsushita, Y. Ishisaki, N.Y. Yamasaki, M. Ishida, T. Ohashi, Type Ia and II supernovae contributions to metal enrichment in the intracluster medium observed with Suzaku. Astrophys. J. 667, 41 (2007). doi:10.1086/522031

S.Y. Sazonov, E.M. Churazov, R.A. Sunyaev, Polarization of resonance X-ray lines from clusters of galaxies. Mon. Not. R. Astron. Soc. 333, 191-201 (2002). doi:10.1046/j.1365-8711.2002.05390.x

S.Y. Sazonov, J.P. Ostriker, R.A. Sunyaev, Quasars: The characteristic spectrum and the induced radiative heating. Mon. Not. R. Astron. Soc. 347, 144-156 (2004). doi:10.1111/j.1365-2966.2004.07184.x

S.Y. Sazonov, J.P. Ostriker, L. Ciotti, R.A. Sunyaev, Radiative feedback from quasars and the growth of massive black holes in stellar spheroids. Mon. Not. R. Astron. Soc. 358, 168-180 (2005). doi:10.1111/ j.1365-2966.2005.08763.x

P. Schuecker, A. Finoguenov, F. Miniati, H. Böhringer, U.G. Briel, Probing turbulence in the Coma galaxy cluster. Astron. Astrophys. 426, 387-397 (2004). doi:10.1051/0004-6361:20041039

K.M. Schure, A.R. Bell, L. O’C Drury, A.M. Bykov, Diffusive shock acceleration and magnetic field amplification. Space Sci. Rev. 173, 491-519 (2012). doi:10.1007/s11214-012-9871-7

C. Shang, S.P. Oh, Disentangling resonant scattering and gas motions in galaxy cluster emission line profiles. Mon. Not. R. Astron. Soc. 433, 1172-1184 (2013). doi:10.1093/mnras/stt790

A. Simionescu, S.W. Allen, A. Mantz, N. Werner, Y. Takei, R.G. Morris, A.C. Fabian, J.S. Sanders, P.E.J. Nulsen, M.R. George, G.B. Taylor, Baryons at the edge of the X-ray-brightest galaxy cluster. Science 331, 1576 (2011). doi:10.1126/science.1200331

A. Simionescu, N. Werner, O. Urban, S.W. Allen, A.C. Fabian, J.S. Sanders, A. Mantz, P.E.J. Nulsen, Y. Takei, Large-scale motions in the Perseus galaxy cluster. Astrophys. J. 757, 182 (2012). doi:10.1088/ 0004-637X/757/2/182

A. Stroe, R.J. van Weeren, H.T. Intema, H.J.A. Röttgering, M. Brüggen, M. Hoeft, Discovery of spectral curvature in the shock downstream region: CIZA J2242.8+5301. Astron. Astrophys. 555, 110 (2013). doi:10.1051/0004-6361/201321267

G. Tabor, J. Binney, Elliptical galaxy cooling flows without mass drop-out. Mon. Not. R. Astron. Soc. 263, 323 (1993)

P.A. Thomas, A.C. Fabian, K.A. Arnaud, W. Forman, C. Jones, The prevalence of cooling flows in early-type galaxies. Mon. Not. R. Astron. Soc. 222, 655-672 (1986)

E.R. Tittley, M. Henriksen, Cluster mergers, core oscillations, and cold fronts. Astrophys. J. 618, 227-236 (2005). doi:10.1086/425952

R.A. Treumann, Fundamentals of collisionless shocks for astrophysical application, 1. Non-relativistic shocks. Astron. Astrophys. Rev. 17, 409-535 (2009). doi:10.1007/s00159-009-0024-2

T. Tsujimoto, K. Nomoto, Y. Yoshii, M. Hashimoto, S. Yanagida, F.K. Thielemann, Relative frequencies of type Ia and type II supernovae in the chemical evolution of the galaxy, LMC and SMC. Mon. Not. R. Astron. Soc. 277, 945 (1995)

V. Vacca, M. Murgia, F. Govoni, L. Feretti, G. Giovannini, E. Orrú, A. Bonafede, The intracluster magnetic field power spectrum in Abell 665. Astron. Astrophys. 514, 71 (2010). doi:10.1051/0004-6361/ 200913060

R.J. van Weeren, H.J.A. Röttgering, M. Brüggen, M. Hoeft, Particle acceleration on megaparsec scales in a merging galaxy cluster. Science 330, 347 (2010). doi:10.1126/science.1194293

R.J. van Weeren, H.J.A. Röttgering, D.A. Rafferty et al., First LOFAR observations at very low frequencies of cluster-scale non-thermal emission: The case of Abell 2256. Astron. Astrophys. 543, 43 (2012). doi:10.1051/0004-6361/201219154 
E. Vangioni, J. Silk, K.A. Olive, B.D. Fields, Cosmic chemical evolution with an early population of intermediate-mass stars. Mon. Not. R. Astron. Soc. 413, 2987 (2011). doi:10.1111/j.1365-2966.2011. 18372.x

F. Vazza, C. Ferrari, A. Bonafede, M. Brüggen, C. Gheller, R. Braun, S. Brown, Filaments of the radio cosmic web: Opportunities and challenges for SKA, in Proceedings of Advancing Astrophysics with the Square Kilometre Array PoS(AASKA14)105 (2015)

A. Vikhlinin, M. Markevitch, S.S. Murray, A moving cold front in the intergalactic medium of A3667. Astrophys. J. 551, 160-171 (2001a). doi:10.1086/320078

A. Vikhlinin, M. Markevitch, S.S. Murray Chandra, Estimate of the magnetic field strength near the cold front in A3667. Astrophys. J. Lett. 549, 47-50 (2001b). doi:10.1086/319126

H.J. Völk, F.A. Aharonian, D. Breitschwerdt, The nonthermal energy content and gamma-ray emission of starburst galaxies and clusters of galaxies. Space Sci. Rev. 75, 279-297 (1996). doi:10.1007/ BF00195040

P. von Ballmoos, J. Alvarez, N. Barrière et al., A DUAL mission for nuclear astrophysics. Exp. Astron. 34, 583-622 (2012). doi:10.1007/s10686-011-9286-6

N. Werner, H. Böhringer, J.S. Kaastra, J. de Plaa, A. Simionescu, J. Vink, XMM-Newton high-resolution spectroscopy reveals the chemical evolution of M 87. Astron. Astrophys. 459, 353 (2006a). doi:10. 1051/0004-6361:20065678

N. Werner, J. de Plaa, J.S. Kaastra, J. Vink, J.A.M. Bleeker, T. Tamura, J.R. Peterson, F. Verbunt, XMM-Newton spectroscopy of the cluster of galaxies 2A 0335+096. Astron. Astrophys. 449, 475 (2006b). doi:10.1051/0004-6361:20053868

N. Werner, F. Durret, T. Ohashi, S. Schindler, R.P.C. Wiersma, Observations of metals in the intra-cluster medium. Space Sci. Rev. 134, 337 (2008). doi:10.1007/s11214-008-9320-9

N. Werner, I. Zhuravleva, E. Churazov, A. Simionescu, S.W. Allen, W. Forman, C. Jones, J.S. Kaastra, Constraints on turbulent pressure in the X-ray haloes of giant elliptical galaxies from resonant scattering. Mon. Not. R. Astron. Soc. 398, 23-32 (2009). doi:10.1111/j.1365-2966.2009.14860.x

N. Werner, A. Simionescu, E.T. Million, S.W. Allen, P.E.J. Nulsen, A. von der Linden, S.M. Hansen, H. Böhringer, E. Churazov, A.C. Fabian, W.R. Forman, C. Jones, J.S. Sanders, G.B. Taylor, Feedback under the microscope-II. Heating, gas uplift and mixing in the nearest cluster core. Mon. Not. R. Astron. Soc. 407, 2063-2074 (2010). doi:10.1111/j.1365-2966.2010.16755.x

N. Werner, O. Urban, A. Simionescu, S.W. Allen, A uniform metal distribution in the intergalactic medium of the Perseus cluster of galaxies. Nature 502, 656-658 (2013). doi:10.1038/nature12646

H. Xu, S.M. Kahn, J.R. Peterson, E. Behar, F.B.S. Paerels, R.F. Mushotzky, J.G. Jernigan, A.C. Brinkman, K. Makishima, High-resolution observations of the elliptical galaxy NGC 4636 with the reflection grating spectrometer on board XMM-Newton. Astrophys. J. 579, 600-606 (2002). doi:10.1086/342828

H. Xu, H. Li, D.C. Collins, S. Li, M.L. Norman, Evolution and distribution of magnetic fields from active galactic nuclei in galaxy clusters. I. The effect of injection energy and redshift. Astrophys. J. 725, 21522165 (2010). doi:10.1088/0004-637X/725/2/2152

H. Xu, H. Li, D.C. Collins, S. Li, M.L. Norman, Evolution and distribution of magnetic fields from active galactic nuclei in galaxy clusters. II. The effects of cluster size and dynamical state. Astrophys. J. 739, 77 (2011). doi:10.1088/0004-637X/739/2/77

H. Xu, F. Govoni, M. Murgia, H. Li, D.C. Collins, M.L. Norman, R. Cen, L. Feretti, G. Giovannini, Comparisons of cosmological magnetohydrodynamic galaxy cluster simulations to radio observations. Astrophys. J. 759, 40 (2012). doi:10.1088/0004-637X/759/1/40

I.V. Zhuravleva, E.M. Churazov, S.Y. Sazonov, R.A. Sunyaev, W. Forman, K. Dolag, Polarization of X-ray lines from galaxy clusters and elliptical galaxies-A way to measure the tangential component of gas velocity. Mon. Not. R. Astron. Soc. 403, 129-150 (2010). doi:10.1111/j.1365-2966.2009.16148.x

I.V. Zhuravleva, E.M. Churazov, S.Y. Sazonov, R.A. Sunyaev, K. Dolag, Resonant scattering in galaxy clusters for anisotropic gas motions on various spatial scales. Astron. Lett. 37, 141-153 (2011). doi:10. 1134/S1063773711010087

I. Zhuravleva, E. Churazov, R. Sunyaev, S. Sazonov, S.W. Allen, N. Werner, A. Simionescu, S. Konami, T. Ohashi, Resonant scattering in the Perseus cluster: Spectral model for constraining gas motions with Astro-H. Mon. Not. R. Astron. Soc. 435, 3111-3121 (2013). doi:10.1093/mnras/stt1506

I. Zhuravleva, E. Churazov, A.A. Schekochihin, S.W. Allen, P. Arévalo, A.C. Fabian, W.R. Forman, J.S. Sanders, A. Simionescu, R. Sunyaev, A. Vikhlinin, N. Werner, Turbulent heating in galaxy clusters brightest in X-rays. Nature 515, 85-87 (2014). doi:10.1038/nature13830

J.A. ZuHone, M. Markevitch, R.E. Johnson, Stirring up the pot: Can cooling flows in galaxy clusters be quenched by gas sloshing? Astrophys. J. 717, 908-928 (2010). doi:10.1088/0004-637X/717/2/908

J.A. ZuHone, M. Markevitch, D. Lee, Sloshing of the magnetized cool gas in the cores of galaxy clusters. Astrophys. J. 743, 16 (2011). doi:10.1088/0004-637X/743/1/16 
J.A. ZuHone, M. Markevitch, G. Brunetti, S. Giacintucci, Turbulence and radio mini-halos in the sloshing cores of galaxy clusters. Astrophys. J. 762, 78 (2013a). doi:10.1088/0004-637X/762/2/78

J.A. ZuHone, M. Markevitch, M. Ruszkowski, D. Lee, Cold fronts and gas sloshing in galaxy clusters with anisotropic thermal conduction. Astrophys. J. 762, 69 (2013b). doi:10.1088/0004-637X/762/2/69

J.A. ZuHone, M.W. Kunz, M. Markevitch, J.M. Stone, V. Biffi, The effect of anisotropic viscosity on cold fronts in galaxy clusters. Astrophys. J. 798, 90 (2015). doi:10.1088/0004-637X/798/2/90 